\title{
Über den Mehrwert organisierter Nachbarschaftshilfe - dargestellt am Beispiel des Zeit-Hilfs-Netzes Steiermark
}

\author{
Tatjana Fischer $^{1}$ (D) Paul Himmelbauer ${ }^{1} \cdot$ Markus Jobst $^{2}$
}

Eingegangen: 16. Oktober 2016 / Angenommen: 25. September 2017 / Online publiziert: 24. Oktober 2017

(C) The Author(s) 2017. Dieser Artikel ist eine Open-Access-Publikation.

Zusammenfassung In diesem Beitrag wird am Beispiel des Zeit-Hilfs-Netzes Steiermark gezeigt, welchen Mehrwert eine auf dem Prinzip der Zeitbank aufbauende, organisierte Nachbarschaftshilfe für die Lebensqualität der teilnehmenden Personen entfaltet und welchen Beitrag sie im Rahmen der Alltagsorganisation verschiedener Personengruppen leistet. Den Ausgangspunkt des Beitrags bilden die Erkenntnisse aus der international geführten Diskussion um die Wirkungsorientierung von Zeitbanken, denen tendenziell große Bedeutung für die soziale Inklusion, die Festigung der Gemeinschaft, aber auch die Abmilderung der Effekte ökonomischer Benachteiligung zugeschrieben wird. Inwiefern das auch auf das seit dem Jahr 2011 in Österreich existierende Zeit-Hilfs-Netz Steiermark zutrifft, wird quantitativ anhand der Mitglieder-, Angebotsund Nachfrageinserate untersucht. Die flankierende Befragung der für das Netz zuständigen Mitarbeiter der Landentwicklung Steiermark sowie der Koordinatoren der lokalen Zeitbankgruppen und die Auswertung des aktuellsten Ergebnisprotokolls des Netzwerktreffens erlauben es, die

Dr. Tatjana Fischer

tatjana.fischer@boku.ac.at

Paul Himmelbauer

paul.himmelbauer@boku.ac.at

Dr. Markus Jobst

markus@jobstmedia.at

1 Department für Raum, Landschaft und Infrastruktur, Institut für Raumplanung, Umweltplanung und Bodenordnung, Universität für Bodenkultur Wien, Peter-Jordan-Straße 82, 1190 Wien, Österreich

2 Department für Geodäsie und Geoinformation, Technische Universität Wien, Erzherzog-Johann-Platz 1/120-6, 1040 Wien, Österreich qualitative Bedeutung des Netzes einzuschätzen. Die empirischen Befunde zeigen, dass im Zeit-Hilfs-Netz Steiermark tendenziell Frauen und Personen im Alter von 50 bis 69 Jahren engagiert sind, die entfaltete Tauschaktivität jedoch quantitativ unbedeutend ist. Gleichwohl werden in den dezentral organisierten Zeit-Hilfs-Netz-Gruppen einzelfallbezogene Beiträge zur Erleichterung des Alltags und zur Lebensqualität der sich aktiv Beteiligenden geleistet. Der gesellschaftliche Mehrwert hingegen bestimmt sich vorrangig aus dem Engagement in den von den Gruppen organisierten gemeinschaftlichen Tätigkeiten. Damit das ZeitHilfs-Netz Steiermark (auch) in Zukunft als flankierendes Angebot in ausgewählten Bereichen der Daseinsvorsorge bestehen kann, braucht es stabile Basisinfrastrukturen auf kommunaler Ebene, eine kontinuierliche professionelle Begleitung und ein klares Leistungsportfolio.

Schlüsselwörter Zeit-Hilfs-Netz Steiermark · Österreich · Zeitbanken · Lebensqualität · Versorgungssicherheit · Organisierte Nachbarschaftshilfe

\section{The Added Value of Organized Neighbourhood Assistance - Illustrated by the Example of the Zeit-Hilfs-Netz Steiermark}

Abstract This paper discusses the added value of time
banks in the sense of organized neighbourhood assistance
using the example of the Styrian time bank called Zeit-
Hilfs-Netz Steiermark for the quality of life of the par-
ticipants and its contribution to everyday life of various
population groups. The theoretical backdrop of this con-
tribution is formed by the findings from the international
discussion on the impact orientation of time banks that as-
cribe to them a certain importance for social inclusion, to 
strengthen communities and to mitigate the negative effects of economic deprivation. This paper investigates if and to what extent the findings for the Zeit-Hilfs-Netz Steiermark (founded in 2011) fit in the international results. For this purpose, data on membership, offer advertisements and on demand as well as the latest network meeting result protocol have been analysed. Additionally, the responsible staff members of the Landentwicklung Steiermark as well as the coordinators of the local time bank groups have been surveyed. The analysis of the result protocol of the latest network meeting confirms the validity of the subjective estimations of the experts. Empirical data show that women and people aged 50 to 69 years form the backbone of the Zeit-Hilfs-Netz Steiermark. Barter activities are less important than joint activities and there is no reciprocity or rather mutual exchange. Nevertheless, this time bank has positive case-by-case effects on the subjective quality of life of the (active) participants. The future existence of the Zeit-HilfsNetz Steiermark and its impacts on securing livelihoods is closely tied to stable basic infrastructures at the local level, professional support and a clear portfolio.

Keywords Zeit-Hilfs-Netz Steiermark · Austria · Time banks · Quality of life - Security of supply · Organized neighbourhood assistance

\section{Einleitung}

Die Transformation des Staates weg vom Sozial- hin zum Gewährleistungsstaat (Kersten 2006: 252) sowie der demographische und soziale Wandel zwingen auch hoch entwickelte Staaten zur Auseinandersetzung mit Fragen zur Aufrechterhaltung der Lebensqualität ${ }^{1}$ und Versorgungssicherheit ${ }^{2}$ der Bevölkerung in sich disparat entwickelnden Räumen (ARL 2016: 2). Überall dort, wo sich aufgrund nicht mehr gegebener wirtschaftlicher Tragfähigkeiten klassische Angebotsformen der Nahversorgung (Geschäfte, Praxen, ÖPNV) aus der Fläche zurückziehen und das Erfordernis der Konzentration von Dienstleistungen in zentralen Orten weite Versorgungswege nach sich zieht, ist die Suche nach und die Entwicklung von adäquaten Alternativen in vollem Gange (BMVBS/BBSR 2011: 5).

\footnotetext{
${ }_{1}$ Unter Lebensqualität wird in diesem Beitrag ein multidimensionales Konstrukt verstanden, welches sich aus der Güte der Passung der individuellen Möglichkeiten und Ansprüche eines Menschen und dessen objektiven (Lebens-)Bedingungen bestimmt (vgl. Post 2014: $170 \mathrm{ff}$.).

${ }^{2}$ Die Autoren dieses Beitrags verstehen unter Versorgungssicherheit die unabhängig vom Wandel der Nahversorgungsstrukturen und individuellen Möglichkeiten im Lebensverlauf gewährleistete Verfügbarkeit von Gütern und Dienstleistungen des täglichen Bedarfs.
}

In diesem Kontext wurde die Bedeutung gut funktionierender Nachbarschaften und der Ressource Zeit (wieder-)erkannt (nef 2008: 10). Seither wird bürgerschaftliche Verantwortung intensiv diskutiert - nicht nur, um finanzielle Lücken zu füllen, die vor allem in ländlichen bzw. strukturschwachen Regionen klaffen, sondern auch, um die Dorfgemeinschaften von mehr oder weniger unverbindlichen Nachbarschaften in verlässlich organisierte Netzwerke umzuwandeln, um diesen einige Teilbereiche der Daseinsvorsorge zu übertragen (BMVI 2015: 120). Die Nachbarschaftsgemeinde bzw. die sorgende Gemeinschaft als soziales Netzwerk soll somit weit über die gegenseitige Unterstützung im Krisenfall hinausgehen und als Koproduzentin bei der Bewältigung des Alltags und der gemeinsamen Freizeitgestaltung fungieren (vgl. Schmid 2009: 74; BMVI 2015: 105 f.).

Gleichzeitig besteht angesichts der Überlagerung der Dynamiken des demographischen Wandels, der Abwanderung und der anhaltenden Ausdünnung lokaler Infrastrukturen (vgl. Alisch/Kümpers 2015: 9) sowie angesichts der zunehmenden Individualisierung und Heterogenisierung der Lebenslagen und Lebensverläufe Sorge über den Aufbau und die Stabilität sozialer Netzwerke (vgl. Pacione 1997: 423 f.; Dehne/Neubauer 2014: 3 f.). Der (neuen) Unübersichtlichkeit und Unklarheit über die Zuständigkeiten der Zivilgesellschaft entspringen neue Anforderungen an die Steuerungs- und Moderationsfunktion der staatlichen Verwaltung (vgl. Steinführer 2015: 5). Unter den gegebenen Bedingungen sind ehrenamtliche Angebote mittelfristig als flankierende Maßnahmen zur Sicherung der Lebensqualität und Versorgung und nicht als Substitut staatlicher Daseinsvorsorge zu interpretieren (vgl. Steinführer 2015: 15), die auf kleinräumiger Ebene gut organisiert sein müssen (BMVI 2015: 120).

In diesem Beitrag werden am Beispiel des Zeit-HilfsNetzes Steiermark (ZHN) die Potenziale und Restriktionen einer Zeitbank als mögliches Organisationsmodell für freiwilliges bürgerschaftliches Engagement - definiert als ,sich selbst auferlegte Verpflichtung“ (More-Hollerweger/ Sprajcer 2009: 4) und als organisatorisches Modell, „die Geschicke des Gemeinwesens“ (Simsa 2001: 44) mitzubestimmen - kritisch diskutiert.

Da der Erfolg von auf Zeittausch basierender, organisierter Nachbarschaftshilfe in Form von Zeitbanken in hohem Maße von der Zufriedenheit der involvierten Personen abhängt (Slay 2011: 5), ist eine kontextbezogene evidenzbasierte Evaluierung des Mehrwerts organisierter Nachbarschaftshilfe für die Lebensqualität der Teilnehmenden und deren konkreter Beitrag zur Daseinsvorsorge aufgrund des Mangels an belastbaren empirischen Daten dringend geboten. Hier setzt der vorliegende Beitrag an: Er stellt die Grundprinzipien und Funktionsweise von Zeitbanken vor und ordnet die Zeitbanken in Bezug auf deren Bedeutung 
für die Daseinsvorsorge international ein. Daran anschließend werden am Beispiel des Zeit-Hilfs-Netzes Steiermark die quantitativen und qualitativen Wirkungen dieser Art organisierter Nachbarschaftshilfe für die hierin involvierten Personen herausgearbeitet und die Ergebnisse vor dem Hintergrund infrastruktureller und demographischer Veränderungen diskutiert.

\section{Zielstellung des Beitrags}

Das Ziel dieses Beitrags ist es, im breiten Themenfeld der Verlagerung von Teilaspekten der Daseinsvorsorge in Richtung bürgerschaftliches Engagement das System der Zeitbanken anhand des Zeit-Hilfs-Netzes Steiermark qualitativ und quantitativ aufzuarbeiten. Die von Blüml (2015) erarbeiteten Ergebnisse zum Zeit-Hilfs-Netz Steiermark regten die Autoren dieses Beitrags an, sich mit Zeitbanken als auf dem Tauschgedanken aufbauende, organisierte Form von Nachbarschaftshilfe vertiefend auseinanderzusetzen. Aufbauend auf Blümls Feststellungen zu den geschlechtsspezifischen Unterschieden hinsichtlich Beteiligung sowie Angebot und Nachfrage (Blüml 2015: 74 ff.), der Identifikation der Pkw-Fahrtüchtigkeit als zentrale Voraussetzung, um aktiv helfen zu können, sowie der Vermutung, dass disperse Siedlungsstrukturen und Defizite in der kommunalen Basisinfrastruktur (u. a. Geschäfte und sozialmedizinische Angebote) die Anbietenden bzw. Helfenden bei der Erbringung von Unterstützungsleistungen behindern (Blüml 2015: $93 \mathrm{f}$.), werden hier folgende querschnittsorientierte Fragen untersucht: ${ }^{3}$

- Was lässt sich aus der Analyse der Mitglieder- und Inseratenstruktur in Bezug auf den Zusammenhang von demographischen Charakteristika der am Zeit-Hilfs-Netz Steiermark Teilhabenden und den Leistungsbereichen ableiten?

- Wie ist es um die Passfähigkeit von Angebot und Nachfrage, die Reziprozität sowie die Intensität der Tauschbeziehungen bestellt?

- Inwiefern profitieren die Teilhabenden in Bezug auf ihre Lebensqualität?

- Welchen Beitrag können Zeitbanken im Kontext der Daseinsvorsorge leisten?

Nicht Gegenstand dieses Beitrags ist jedoch der Vergleich unterschiedlicher Formen bzw. Modelle bürgerschaftlicher Beteiligung und deren Beiträge zur Sicherung der Daseinsvorsorge wie der aktuell intensiv diskutierten Bürger-, Sozial- oder Seniorengenossenschaften (vgl. El-

\footnotetext{
${ }^{3}$ Die hier vorgestellten Ergebnisse wurden eigens für diesen Beitrag generiert. Sie entstanden ausschließlich im Rahmen der Eigenforschung.
}

sen 2012; BMVI 2015; Schmale 2017) mit dem Modell Zeitbank.

\section{Zeitbanken - eine internationale Einordnung des Themas}

Organisierte Nachbarschaftshilfe folgt dem Selbsthilfeprinzip und basiert auf dem bargeldlosen Leistungs- und Warentausch zwischen den Teilnehmenden (Hiller 2013: 120). Das Tauschmittel bzw. die Währung, in der Leistungen verrechnet werden, ist die Zeit. Die Idee zu sogenannten komplementären Währungen bzw. Local Exchange and Trading Systems (LETS) zum Aufbau solidarischer Wirtschaftssysteme (für benachteiligte Personengruppen) hatte Anfang der 1980er-Jahre der Kanadier Micheal Linton (Hiller 2013: 120). Um den Konsequenzen der bereits in den 1980erJahren in den USA beobachtbaren sozialen Erosionsprozessen entgegenzuwirken, entwickelte auch der Amerikaner Edgar Cahn (Seyfang 2002: 2; Hiller 2013: 123) sogenannte Zeitbanken, die die älteste, in unterschiedlichen Formen weltweit verbreitete dienstleistungsbasierte Lokalwährung darstellen (Shih/Bellotti/Han et al. 2015: 1075).

Zeitbanken wird seither ein hohes Potenzial in Bezug auf den Aufbau von Gemeinschaften und die Reanimation von Nachbarschaftshilfe zugeschrieben (Ozanne 2010: 4; Shih/Bellotti/Han et al. 2015). Begründet wird dies durch folgende Grundprinzipien und Merkmale von Zeitbanken:

- die Freiwilligkeit der Teilhabe (Gegenleistungen sind nicht einklagbar),

- den bargeldlosen Leistungstausch,

- die Gleichwertigkeit der erbrachten Leistung (Seyfang 2003a: 700; Lasker/Collom/Bealer et al. 2011: 103),

- die indirekte Reziprozität, das heißt die Wechselseitigkeit von Geben und Nehmen (Yamagishi/Cook 1993: 235) und

- das Offensein für alle an einer Teilhabe interessierten Personen (Seyfang 2002: 6).

In der Regel ist die Tauscheinheit die Stunde.

Welche Erwartungen an Zeitbanken thematisch bzw. im Kontext der Organisation von Daseinsvorsorge geknüpft werden, hängt von den spezifischen nationalen politischen, ökonomischen und sozialen Rahmenbedingungen und dem anbietbaren Portfolio ab (vgl. Peacock 2000: 57; Boyle 2014: 6). Unterschiede bestehen auch darin, ob Zeitbanken von lokalen Organisationen geführt werden, Multiplikatoren als Mitglieder in Zeitbanken fungieren oder der direkte Tausch zwischen Einzelpersonen im Vordergrund steht (Shih/Bellotti/Han et al. 2015: 1077). Somit kann eine etablierte Zeitbank (theoretisch) auf systemischer Ebene eine Koproduzentin in der Daseinsvorsorge sein (nef 2008: 3; Shih/Bellotti/Han et al. 2015: 1076) und Mehrwerte auf der 
persönlichen Ebene der teilnehmenden Personen entfalten: Sei es, um das Bedürfnis zu helfen zu befriedigen, sei es, um sich als Teil einer Community zu fühlen (Thorne 1996: 1364).

Obwohl Zeitbanken in der Fachliteratur tendenziell als gute Lösung zur Kompensation infrastruktureller Defizite präsentiert werden, muss auch auf deren Grenzen hingewiesen werden: Zeitbanken haben einen klein(st)räumigen Wirkungsbereich und deren Erfolg ist abhängig von der Stabilität des Engagements und der Hilfsbeziehungen sowie den Fertigkeiten und der altersstrukturellen Zusammensetzung der sich aktiv Beteiligenden (Seyfang 2003b). Darüber hinaus besteht weder für die Anbietenden noch für die Nachfragenden ein Anspruch auf Leistung (Fraaß/Görtler/ Rosenkranz 2016: 9). Zudem gefährdet das Fehlen einer entsprechenden Nachfrage die Kontinuität des Angebots. Shih, Bellotti, Han et al. (2015: $1078 \mathrm{ff}$.) identifizierten in diesem Zusammenhang im Rahmen einer Auswertung der drei größten Zeitbanken in den USA die mangelnde Erreichbarkeit von Angeboten (vor allem in ländlichen Regionen) als relevantes Moment, weiters Schwierigkeiten in der zeitlichen Koordination des Austauschs sowie Fragen der Verfügbarkeit von (vor allem höher qualifizierten) Angeboten. Auch der Mangel an Unterstützung durch Organisationen bzw. staatliche Stellen schränkten die Verbreitung von Zeitbanken ein.

\section{Zeitbanken in Österreich}

Im internationalen Vergleich nehmen sich die österreichischen Zeitbanken in quantitativer Hinsicht bescheiden aus. Dies wird vor allem im Hinblick auf die USA deutlich, wo der sogenannte Dritte Sektor ${ }^{4}$ aufgrund der sozial- und gesundheitspolitischen Rahmenbedingungen inzwischen eine tragende Säule der Daseinsvorsorge ist (vgl. Peacock 2000: 61). Laut Höllhumer und Trukeschitz (2016: 13) wurden in Österreich die ersten Zeitbanken im Jahr 2006 gegründet. Eine Bestandsaufnahme aus dem Jahr 2015 zeigt, dass rund 8.000 Personen in den gezählten 40 Tauschkreisen und Zeitbanken registriert sind, es eine räumliche Konzentration in den Bundesländern Oberösterreich, Niederösterreich und der Steiermark gibt, der Bestand an Zeitbanken Fluktuationen unterliegt und die Transaktionen in den Zeitbanken vor allem zwischen Privatpersonen erfolgen (Höllhumer/ Trukeschitz 2016: 12 ff.). Informationen zu den Dimensionen des Angebots und der Nachfrage bzw. zum Umfang oder zur Intensität der Tauschbeziehungen liegen nicht vor.

\footnotetext{
${ }^{4}$ Unter dem Dritten Sektor werden alle Angebote verstanden, die eine Zwischenstellung im Dreieck Staat-Markt-Gemeinschaft einnehmen (Priller/Zimmer 2000: 304).
}

\section{Das Beispiel Zeit-Hilfs-Netz Steiermark}

\subsection{Entstehungsgeschichte und Organisationsstruktur}

Der Verein „Landentwicklung Steiermark“ hat das Ziel, nachhaltige Regionalentwicklung über verschiedene Projekte zu betreiben und dabei die Bürgerinnen und Bürger der Steiermark mit einzubeziehen. ${ }^{5}$ Zwischen 1997 und 2015 war er die Leitstelle für alle Lokalen Agenda-21-Prozesse in der Steiermark und auch mit deren Umsetzung betraut. Er initiierte Bewusstseinsbildungsprozesse und Projektinitiativen. Die Arbeit in den Gemeinden und der Austausch mit den sich in den Lokalen Agenda-21-Prozessen ${ }^{6}$ beteiligenden Personen ließen erkennen, dass (Ab-)Wanderungsdynamiken, altersstrukturelle Verschiebungen, Siedlungsstrukturen und Veränderungen im kommunalen Angebot an Gütern und Diensten des täglichen Bedarfs einander überlagern. Dies hatte eine Lockerung oder Auflösung der sozialen Kohäsion sowie Erschwernisse beim Zugang zu Infrastruktur in den Gemeinden zur Folge.

Der Blick auf die demographische und ökonomische Entwicklung des Bundeslandes Steiermark erklärt den wahrgenommenen Handlungsbedarf, zeigen sich doch abgesehen von der Landeshauptstadt Graz und dem politischen Bezirk Graz-Umgebung alle anderen Räume von Bevölkerungsstagnation oder -schrumpfung betroffen. Bis 2050 werden sich die Disparitäten in Bezug auf die räumliche Verteilung der Bevölkerung weiter verschärfen. Die einzigen Bevölkerungsgewinner werden Graz $(+28,2 \%)$ und Graz-Umgebung $(+12 \%)$ bleiben, und ab 2020 wird der Rückgang von Personen im erwerbsfähigen Alter vor allem in strukturschwachen peripheren Gebieten spürbar werden. Parallel dazu schreitet die demographische Alterung voran. Bis 2050 wird knapp ein Drittel der steirischen Bevölkerung älter als 65 Jahre sein und die Anzahl der über 85-Jährigen wird sich bis dahin etwa verdreifacht haben (Land Steiermark 2016a: 5 ff.). Die raumdifferenzierte Betrachtung des Bruttoinlandsprodukts pro Kopf zeigt, dass die Region Graz mit 44.200 Euro pro Einwohner (Stand 2013) die Spitze des Bundeslandes bildet, während die übrigen steirischen Regionen deutlich unter dem Bundeswert von 38.100 Euro pro Kopf (2013) liegen (Land Steiermark 2016b: 35).

Um diese negativen Entwicklungen abzumildern, beauftragte das Land Steiermark im Jahr 2011 die Landentwicklung Steiermark mit der Konzeption eines auf dem Modell Zeitbank aufbauenden Zeit-Hilfs-Netzes. Der Anspruch

\footnotetext{
5 Vgl. http://www.landentwicklung-steiermark.at/Leitbild (18.08.2017),

${ }^{6}$ Lokale Agenda-21-Prozesse dienen dazu, im Dialog von Bürgern, Verwaltung und Wirtschaft Leitbilder zur nachhaltigen Entwicklung zu formulieren und umzusetzen, wie sie im Jahr 1992 im Rahmen der von den Vereinten Nationen (UN) formulieren Agenda 21 vorgesehen sind.
} 
an das Zeit-Hilfs-Netz Steiermark lag darin, die Nachbarschaftshilfe wiederzubeleben und die Kommunikation innerhalb der heterogener werdenden (Land-)Bevölkerung zu befördern. Zudem wurde in diesem Ansatz eine Möglichkeit gesehen, die Kostenbelastungen der Gemeinden im Bereich sozialer Hilfen zu senken. ${ }^{7}$ Alle interessierten Kommunen waren als Gebietskörperschaften eingeladen, sich daran zu beteiligen. Möglicherweise begründeten auch die offiziellen Zahlen zum ehrenamtlichen Engagement in der Steiermark (Land Steiermark 2010: 5 ff.) die Hoffnungen in das ZeitHilfs-Netz Steiermark. Demnach leistet etwa jeder Zweite bereits Freiwilligenarbeit, die Beteiligungsquote in Bezug auf formelle Tätigkeiten liegt bei $31 \%$, in Bezug auf informelle bei $33 \%$ (BMASK 2015: 23). Sport-, Kulturund Brauchtumsvereine machen knapp $47 \%$ aller Vereine aus, gefolgt von Einsatzorganisationen $(9,5 \%)$ und sonstigen Vereinen $(43,9 \%)$, wobei letztere überwiegend zur Dorfgemeinschaft beitragen (Land Steiermark 2010: $5 \mathrm{ff}$.).

Über Fördermittel des Landes - diese sind mittlerweile ausgelaufen - wurden die mit der Organisation des ZeitHilfs-Netzes Steiermark verbundenen Aufgaben finanziert. Hierzu zählten die Projektbegleitung und Beratung der teilnehmenden Gemeinden, die Kontaktpflege mit den Koordinatoren der Gruppen des Zeit-Hilfs-Netzes sowie die Organisation von landesweiten Netzwerktreffen. Heute bildet der eingetragene Verein "Zeit-Hilfs-Netz Steiermark“ das organisatorische Dach des Netzes, welches dezentral in Form von Gruppen organisiert ist, die nach ihren Standortgemeinden benannt sind. Die Mitglieder des Vereins sind im Rahmen ihrer ehrenamtlichen Tätigkeit unfall- und haftpflichtversichert. Der jährliche Mitgliedsbeitrag von zehn Euro umfasst neben dem Versicherungsschutz auch eine jährliche Gutschrift von fünf Gutstunden auf dem Stundenkonto. Das soll neuen Mitgliedern als Anreiz dienen, nicht nur Unterstützung zu leisten, sondern diese auch in Anspruch zu nehmen. Seit seiner Gründung im Jahr 2011 waren zehn steiermärkische Gemeinden im Zeit-Hilfs-Netz Steiermark aktiv. Mittlerweile sind zwei Gemeinden ausgeschieden, zwei neue hinzugekommen. Zum 31. Dezember 2015 umfasst das Zeit-Hilfs-Netz Steiermark drei ländliche Gemeinden, sechs Kleinstädte und einen Bezirksteil der Landeshauptstadt Graz (vgl. Abbildung 1).

Tabelle 1 zeigt, dass die zum Zeitpunkt der Auswertung im Zeit-Hilfs-Netz Steiermark aktiven Gemeinden hinsichtlich Einwohnerzahl, Demographie und Funktion heterogen sind. Auffallend ist die Spannweite bezüglich der Mitgliedszahlen in den Gruppen. Sie reicht von 11 bis 103 Personen. Ein Zusammenhang zwischen Raumtypus bzw. Größe der Gemeinde und Mitgliederzahl lässt sich nicht herstellen.

\footnotetext{
${ }_{7}$ Mündliche Mitteilung der Landentwicklung Steiermark vom 6. September 2016.
}

Gemeinsam ist den Gruppen, dass deren Mitglieder vorrangig in den namengebenden Standortgemeinden wohnen.

\subsection{Funktionsweise als Marktplatz}

Das Zeit-Hilfs-Netz Steiermark ist als (virtueller) Marktplatz bzw. Kommunikationsplattform konzipiert und bietet tauschbereiten Vereinsmitgliedern die Möglichkeit, Angebote und Nachfragen in verschiedenen Leistungsbereichen in Form von Inseraten sichtbar zu machen. Getauscht werden können Hilfestellungen in den folgenden Leistungsbereichen (Kategorien):

- administrative Hilfen

- Fahrtendienste/Begleitung

- Freizeit/Geselliges

- handwerkliche Hilfen

- Kinderbetreuung

- Hilfe im Haushalt

- Hilfe beim Kochen und Essen

- Gesundheit und Körperpflege ${ }^{9}$

- Selbstgemachtes aus dem Garten

- Secondhandprodukte

Diese Inserate werden sowohl in der Online-Plattform Cyclos - einer international etablierten Kommunikationsplattform (Boyle 2014: 7) - als auch analog in Form von in den Standortgemeinden ausliegenden und vom Organisationsteam laufend aktualisierten, jedoch nicht redaktionell bearbeiteten Listen ${ }^{10}$ verwaltet.

\section{Empirische Untersuchung des Zeit-Hilfs-Netzes Steiermark}

Zur Beantwortung der in Kapitel 2 formulierten Fragestellungen werden drei empirische Zugänge gewählt: die Datenanalyse der Mitglieder- und Inseratendatenbank, die explorative Expertenbefragung von für das Zeit-Hilfs-Netz Steiermark verantwortlichen Personen der Landentwicklung Steiermark und der Leiter der ZHN-Gruppen sowie die Dokumentenanalyse des Ergebnisprotokolls zum jüngsten Netzwerktreffen.

\footnotetext{
${ }^{8}$ Diese Kategorisierung ist an das Vorarlberger Talente-System angelehnt (mündliche Mitteilung der Landentwicklung Steiermark vom 6. September 2016).

9 Im Bereich der Kategorie „Gesundheit und Körperpflege“ dürfen nur Leistungen erbracht werden, die nicht im österreichischen Gesundheits- und Krankenpflegegesetz (GuKG 1997) geregelt sind.

${ }^{10}$ Es wird beispielsweise nicht überprüft, ob die Detailbeschreibungen der angebotenen bzw. nachgefragten Leistungen mit übergeordneten Kategorien übereinstimmen.
} 


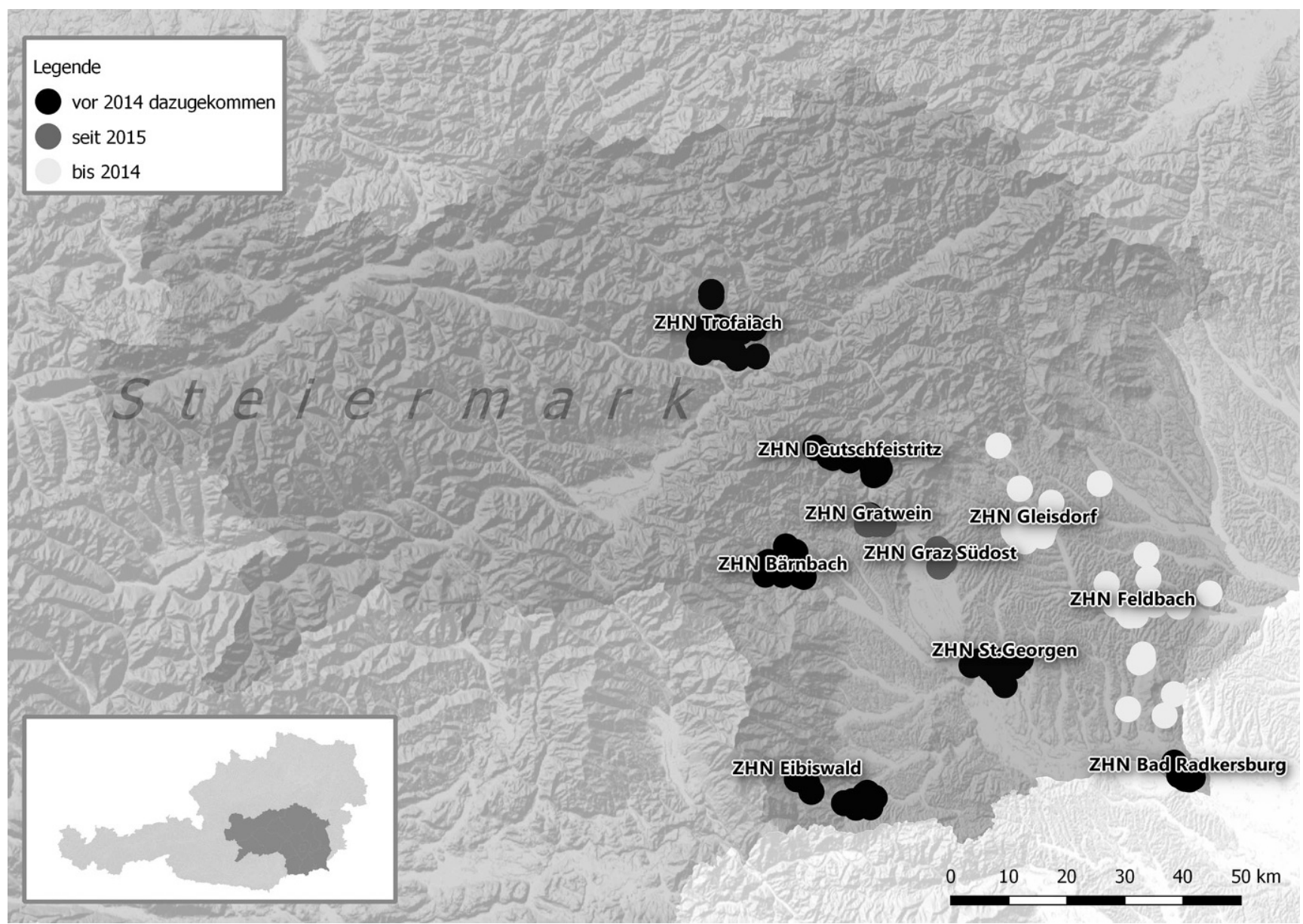

Abbildung 1 Räumliche Verteilung der teilnehmenden Gemeinden

\subsection{Zielstellung der empirischen Zugänge}

Die Datenanalyse dient dazu, einen Überblick über das demographische Profil und die Wohnstandorte der registrierten Mitglieder zu gewinnen, die quantitative Dimension und inhaltliche Struktur von Angebot und Nachfrage zu erfassen sowie die theoretische Passung von angebotenen und nachgefragten Leistungen abzuleiten und etwaige Veränderungen in der Mitglieder- und Angebotsstruktur zu identifizieren. Zweck der explorativen Expertenbefragung ist es, die Motivation für die Teilhabe und damit die Relationen bzw. etwaige Schieflagen von Angebot und Nachfrage im Zeit-Hilfs-Netz Steiermark zu verstehen, die Bedeutung des Zeit-Hilfs-Netz Steiermark im zeitlichen Verlauf zu erklären sowie einen Ausblick auf dessen Weiterentwicklung zu geben. Die im Zeit-Hilfs-Netz Steiermark aktiven Mitglieder wurden zwecks Wahrung ihrer Anonymität nicht befragt.

Um jedoch Informationen zum subjektiven Mehrwert des Zeit-Hilfs-Netz Steiermark für die hierin aktiven Personen, den gesellschaftlichen Mehrwert des Netzes und die Bedeutung des Tauschprinzips ableiten zu können, die Interpre- tation der Ergebnisse der Analyse der Inserate und der Expertenbefragung zu verfeinern sowie den Aspekt etwaiger zeitlicher Schwankungen im Angebot und in der Nachfrage näher zu untersuchen, wurde das Ergebnisprotokoll vom vierten Netzwerktreffen des Zeit-Hilfs-Netzes Steiermark, welches im April 2015 stattgefunden hat, herangezogen. Dieses ist gleichsam eine Zwischenbilanz der bisherigen Aktivitäten, Stolpersteine und Erfolge der ersten fünf Bestandsjahre des Zeit-Hilfs-Netzes Steiermark.

\subsection{Methodische Vorgehensweise}

\subsubsection{Datenanalyse der Mitglieder- und Inseratendatenbank}

Zur inhaltlichen Auswertung wurden anonymisierte Gesamtlisten der Mitglieder seit Gründung des Zeit-Hilfs-Netzes Steiermark im Jahr 2011 sowie Listen der Angebote und Nachfragen zu den Zeitpunkten 31. Dezember 2014 und 31. Dezember 2015 als Excel-Tabellen von der Landentwicklung Steiermark bereitgestellt. Das Datenmanagement - es wurden jährlich zum Stichtag 31. Dezember Anzahl und Art 
Tabelle 1 Profil der Zeit-Hilfs-Netz-Gruppen Steiermark und Mitgliederstruktur Quellen: Statistik Austria (2016a); Statistik Austria (2016b); Land Steiermark (2016a); eigene Berechnungen

\begin{tabular}{|c|c|c|c|c|c|c|c|c|}
\hline \multirow[t]{2}{*}{ ZHN-Gruppe } & \multirow[t]{2}{*}{ Funktion der Gemeinde } & \multirow{2}{*}{$\begin{array}{l}\text { Einwohner } \\
2016\end{array}$} & \multirow[t]{2}{*}{ Altersstruktur } & \multirow{2}{*}{$\begin{array}{l}\text { Bevölkerungs- } \\
\text { veränderung } \\
\text { 2002-2016 }\end{array}$} & \multicolumn{2}{|c|}{ ZHN-Gruppenmitglieder } & \multirow[b]{2}{*}{ Geschlecht } & \multirow[b]{2}{*}{$\begin{array}{l}\text { wohnhaft in ZHN } \\
\text { Gemeinde: an- } \\
\text { derswo }\end{array}$} \\
\hline & & & & & Anzahl & $\begin{array}{l}\text { nach Alters- } \\
\text { gruppe }\end{array}$ & & \\
\hline $\begin{array}{l}\text { Bad Radkersburg (nach } \\
\text { wie vor im ZHN aktiv) }\end{array}$ & $\begin{array}{l}\text { ehemalige Bezirkshauptstadt; Klein- } \\
\text { stadt im ländlichen Raum (regionales } \\
\text { Zentrum) }\end{array}$ & 3.114 & $\begin{array}{l}<20 \mathrm{~J} .: 15 \% \\
\geq 60 \mathrm{~J} .: 35 \%\end{array}$ & $-6,0 \%$ & 13 & $\begin{array}{l}<20 \text { J.: } 0 \\
\geq 60 \text { J.: } 6\end{array}$ & $\begin{array}{l}\mathrm{w}=9 \\
\mathrm{~m}=4\end{array}$ & $12: 1$ \\
\hline $\begin{array}{l}\text { Bärnbach (nach wie vor } \\
\text { im ZHN aktiv) }\end{array}$ & $\begin{array}{l}\text { Kleinstadt im ländlichen Raum (Aus- } \\
\text { pendlergemeinde) }\end{array}$ & 5.697 & $\begin{array}{l}<20 \text { J.: } 19 \% \\
\geq 60 \text { J.: } 28 \%\end{array}$ & $+7,0 \% *)$ & 34 & $\begin{array}{l}<20 \mathrm{~J} .: 1 \\
\geq 60 \mathrm{~J} .: 21\end{array}$ & $\begin{array}{l}\mathrm{w}=22 \\
\mathrm{~m}=11 \\
\mathrm{k} . \mathrm{A} .=1\end{array}$ & $5: 1$ \\
\hline $\begin{array}{l}\text { Deutschfeistritz (nach wie } \\
\text { vor im ZHN aktiv) }\end{array}$ & $\begin{array}{l}\text { dünn besiedelte ländliche Gemeinde } \\
\text { (Auspendlergemeinde) }\end{array}$ & 4.188 & $\begin{array}{l}<20 \mathrm{~J} .: 12 \% \\
\geq 60 \mathrm{~J} .: 24 \%\end{array}$ & $-0,6 \%$ & 17 & $\begin{array}{l}<20 \text { J.: } 0 \\
\geq 60 \text { J.: } 8\end{array}$ & $\begin{array}{l}\mathrm{w}=13 \\
\mathrm{~m}=4\end{array}$ & $3: 1$ \\
\hline $\begin{array}{l}\text { Eibiswald (nach wie vor } \\
\text { im ZHN aktiv) }\end{array}$ & $\begin{array}{l}\text { dünn besiedelte ländliche Gemeinde } \\
\text { (Auspendlergemeinde) }\end{array}$ & 6.590 & $\begin{array}{l}<20 \mathrm{~J} .: 18 \% \\
\geq 60 \mathrm{~J} .: 27 \%\end{array}$ & $-8,5 \%$ & 22 & $\begin{array}{l}<20 \text { J.: } 0 \\
\geq 60 \text { J.: } 13\end{array}$ & $\begin{array}{l}\mathrm{w}=17 \\
\mathrm{~m}=5\end{array}$ & $3: 1$ \\
\hline $\begin{array}{l}\text { Zonta Feldbach (nicht } \\
\text { mehr aktiv) }\end{array}$ & $\begin{array}{l}\text { ehemalige Bezirkshauptstadt; Klein- } \\
\text { stadt im ländlichen Raum (regionales } \\
\text { Zentrum) }\end{array}$ & 13.313 & $\begin{array}{l}<20 \mathrm{~J} .: 20 \% \\
\geq 60 \mathrm{~J} .: 24 \%\end{array}$ & $+5,5 \%$ & 33 & $\begin{array}{l}<20 \mathrm{~J} .: 0 \\
\geq 60 \mathrm{~J} .: 7\end{array}$ & $\begin{array}{l}\mathrm{w}=27 \\
\mathrm{~m}=6\end{array}$ & $1: 1$ \\
\hline $\begin{array}{l}\text { Gleisdorf (nicht mehr } \\
\text { aktiv) }\end{array}$ & $\begin{array}{l}\text { Kleinstadt im ländlichen Raum (regio- } \\
\text { nales Zentrum) }\end{array}$ & 10.452 & $\begin{array}{l}<20 \mathrm{~J} .: 20 \% \\
\geq 60 \mathrm{~J} .: 24 \%\end{array}$ & $+15,7 \%$ & 25 & $\begin{array}{l}<20 \mathrm{~J} .: 0 \\
\geq 60 \mathrm{~J} .: 12\end{array}$ & $\begin{array}{l}\mathrm{w}=19 \\
\mathrm{~m}=6\end{array}$ & $6: 1$ \\
\hline $\begin{array}{l}\text { Gratwein-Straßengel (seit } \\
2015 \text { aktiv) }\end{array}$ & $\begin{array}{l}\text { Kleinstadt im ländlichen Raum (Aus- } \\
\text { pendlergemeinde) }\end{array}$ & 12.806 & $\begin{array}{l}<20 \mathrm{~J} .: 20 \% \\
\geq 60 \mathrm{~J} .: 25 \%\end{array}$ & $+7,7 \% *)$ & 11 & $\begin{array}{l}<20 \text { J.: } 0 \\
\geq 60 \text { J.: } 2\end{array}$ & $\begin{array}{l}\mathrm{w}=8 \\
\mathrm{~m}=3\end{array}$ & $11: 1$ \\
\hline $\begin{array}{l}\text { Graz-Südost (seit } 2015 \\
\text { aktiv) }\end{array}$ & $\begin{array}{l}\text { Teil eines Bezirks der Landeshauptstadt } \\
\text { Graz }\end{array}$ & 14.305 & k.A. & k.A. & 14 & $\begin{array}{l}<20 \text { J.: } 0 \\
\geq 60 \text { J.: } 6\end{array}$ & $\begin{array}{l}\mathrm{w}=10 \\
\mathrm{~m}=4\end{array}$ & $13: 1$ \\
\hline $\begin{array}{l}\text { St. Georgen an der Stie- } \\
\text { fing (nach wie vor im } \\
\text { ZHN aktiv) }\end{array}$ & $\begin{array}{l}\text { dünn besiedelte Gemeinde (Auspendler- } \\
\text { gemeinde) }\end{array}$ & 1.492 & $\begin{array}{l}<20 \mathrm{~J} .: 20 \% \\
\geq 60 \mathrm{~J} .: 25 \%\end{array}$ & $-1,0 \%$ & 38 & $\begin{array}{l}<20 \text { J.: } 5 \\
\geq 60 \text { J.: } 14\end{array}$ & $\begin{array}{l}\mathrm{w}=22 \\
\mathrm{~m}=16\end{array}$ & $12: 1$ \\
\hline $\begin{array}{l}\text { Trofaiach (nach wie vor } \\
\text { im ZHN aktiv) }\end{array}$ & $\begin{array}{l}\text { Kleinstadt im ländlichen Raum (Aus- } \\
\text { pendlergemeinde) }\end{array}$ & 11.227 & $\begin{array}{l}<20 \mathrm{~J} .: 17 \% \\
\geq 60 \mathrm{~J} .: 33 \%\end{array}$ & $-4,2 \%$ & 103 & $\begin{array}{l}<20 \text { J.: } 2 \\
\geq 60 \text { J.: } 39\end{array}$ & $\begin{array}{l}\mathrm{w}=75 \\
\mathrm{~m}=27 \\
\mathrm{k} \cdot \mathrm{A} .=1\end{array}$ & $9: 1 * *)$ \\
\hline
\end{tabular}
*) Bevölkerungszuwachs infolge der Gemeindestrukturreform 2015. **) Im Rahmen der Gemeindestrukturreform wurden der Stadtgemeinde Trofaiach die beiden ehemaligen Gemeinden Hafning
bei Trofaiach und Gai zugeordnet, in welchen zum 31.12.2015 91 der 103 Mitglieder wohnten; der Marktgemeinde Eibiswald wurde u. a. die ehemalige Gemeinde Pitschgau zugeordnet, in welcher zum 31.12.2015 17 der 22 Mitglieder wohnten. 
der Inserate erhoben -, die Art der Datenarchivierung sowie personelle Umstrukturierungen beim Datenbereitsteller begründen die Beschränkung auf den Vergleich der beiden Zeitpunkte 31. Dezember 2014 und 31. Dezember 2015. Die Erwartungen der Autoren dieses Beitrags an den Vergleich der Inserate bezogen sich auf die Identifikation geschlechts- und ZHN-gruppenspezifischer Unterschiede hinsichtlich Art und Menge der Angebote und Nachfragen.

In einem ersten Schritt wurden die drei Datenbanken über die Identifikationsnummer der registrierten Mitglieder miteinander verknüpft. Daran schloss sich eine auf der Auszählung von Häufigkeiten beruhende Analyse der Inhalte dieser drei Datenquellen an, durch die sich folgende Aspekte darstellen lassen:

- das demographische Profil der registrierten Mitglieder über die Merkmale Alter und Geschlecht,

- die Wohnadressen der registrierten Mitglieder,

- die konkrete Absicht der registrierten Mitglieder, Leistungen aktiv zu erbringen und/oder zu empfangen (über die Zuordnung als anbietendes und/oder nachfragendes Mitglied),

- die quantitative Dimension und kategorienbezogene Struktur von Angebot und Nachfrage sowie das Verhältnis von Angebot und Nachfrage (über die Häufigkeit der angebotenen und/oder nachgefragten Leistungsbereiche),

- die differenzierte, kategorienbezogene Darstellung des Leistungsspektrums (über die zusätzlichen Detailangaben zu den von den Mitgliedern angebotenen und nachgefragten Hilfestellungen),

- die kategorienbezogenen Unterschiede in der Angebotsund Nachfragestruktur zu den definierten zwei Zeitpunkten.

Die theoretische Passung von Angebot und Nachfrage wurde wie folgt konstruiert: Zuerst wurden die Detailbeschreibungen der Angebote und Nachfragen getrennt nach Kategorien bzw. Leistungsbereichen und ZHN-Gruppen für den jeweils aktuell verfügbaren Zeitpunkt ${ }^{11}$ auf Hinweise zu den zeitlichen und räumlichen Bedingungen des Angebots und den Besonderheiten der Nachfrage hin untersucht. Die Auseinandersetzung der zeitlichen Dimension dient der Klärung, ob die Leistungen mitgliederbezogen und kategorienbezogen wiederkehrend angeboten bzw. nachgefragt werden. Zur Darstellung der zeitbezogenen Rhythmik wurden a priori zwei Ausprägungen festgelegt: unregelmäßig und regelmäßig. In weiterer Folge wurden alle Detailbeschreibungen der Angebots- und Nachfrageinserate auf zeitbezogene Hinweise überprüft. Der Ausprägung ,,unregelmäßige Hilfen“ wurden alle Inserate zugeordnet, die verba-

${ }^{11}$ Für die beiden ZHN-Gruppen Gleisdorf und Zonta Feldbach ist dies der 31. Dezember 2014. le Beschreibungen enthalten, die explizit die Unregelmäßigkeit des Angebots bzw. der Nachfrage zum Ausdruck bringen wie etwa ,ab und zu“, „,bei Gelegenheit/gelegentlich“, ,im Bedarfsfall“, ,im Notfall“ oder ,,spontan“; der Ausprägung ,,regelmäßige Hilfen“ wiederum alle, in denen der Begriff ,regelmäßig“ vorkommt. Aus der Analyse der räumlichen Reichweite, das heißt des Aktionsradius der Anbietenden bzw. der aus der Sicht der Nachfragenden erforderlichen Aktionsradien, sollten die Fahraufwände abgeleitet werden. Diese räumliche Anforderung wurde wie folgt codiert: „1“ für Reichweite bzw. Aktionsradius innerhalb der Wohngemeinde der Anbietenden bzw. Nachfragenden, ,2“ für inner- und außerhalb der Wohngemeinde, ,3“ für außerhalb der Wohngemeinde. Nicht eindeutig zuordenbare Angaben erhielten den Wert „4“ (Aktionsradius unbestimmt).

Danach wurden die neucodierten Daten in einer Datenbank zusammengeführt und über die kategorienbezogene, zeitliche und räumliche Kongruenz alle theoretischen Tauschpaare (Tandems) auf der Ebene der ZHN-Gruppen ermittelt. Abschließend wurde der zeitliche Aufwand für die theoretisch möglichen Tauschakte festgestellt. Durch die Berechnung der Entfernungen zwischen den Wohnadressen der Tandempartner über Google Maps wurde der zeitliche Aufwand der Distanzüberwindung mit dem Auto bzw. die erforderliche Gehzeit bestimmt. Der Umfang von im ZeitHilfs-Netz Steiermark bereits geleisteten Stunden, die Intensität der Tauschbeziehung(en) und die Abschätzung der Reziprozität der Tauschbeziehungen im Netz, wie sie von Fraňková, Fousek, Kala et al. (2014: 268) im Jahr 2013 an einem tschechischen Beispiel gezeigt worden ist, ließen sich auf der Basis der verfügbaren Daten jedoch nicht abbilden.

\subsubsection{Explorative Expertenbefragung}

Im Frühjahr 2016 wurden die mit der Organisation des ZeitHilfs-Netzes Steiermark betrauten Mitarbeiter der Landentwicklung Steiermark sowie alle Gruppenleiter mittels schriftlicher Befragung um eine Reflexion der Ergebnisse der Mitglieder- und Inseratenanalyse, der Bestimmungsfaktoren der Teilhabe der Personen, eine Einschätzung des aktuellen Beitrages des Zeit-Hilfs-Netzes Steiermark zur Schließung von kommunalen Versorgungslücken sowie um einen Ausblick auf die nähere Zukunft des Netzes ersucht. Der Fragebogen für die Gruppenleiter umfasste 23 offene Fragen sowie ein Textfeld für Anmerkungen und wurde von der Landentwicklung Steiermark an die zehn Gruppenleiter per E-Mail übermittelt. Fünf Gruppenleiter nahmen an der Befragung teil. Der Fragenkatalog für die Mitarbeiter der Landentwicklung Steiermark enthielt dieselben Fragen sowie zwei weitere zu den Hintergründen für das Ausscheiden bzw. Neuhinzukommen von ZHN-Gruppen. Die Antworten wurden thematisch gegliedert, nach der Methode des stän- 
digen Vergleichens nach Glaser und Strauss (1998) synthetisiert und mittels qualitativer Inhaltsanalyse nach Mayring (2002) ausgewertet.

\subsubsection{Dokumentenanalyse des Ergebnisprotokolls zum vierten Netzwerktreffen des Zeit-Hilfs-Netzes Steiermark}

Das 13-seitige Fotoprotokoll des vierten Netzwerktreffens des Zeit-Hilfs-Netzes Steiermark fasst die Erfahrungen der im Zeit-Hilfs-Netz Steiermark engagierten Personen und deren Bewertung von Erfolg und Mehrwert des Netzes für die Lebensqualität zusammen. Insgesamt wurden im $\mathrm{Zu}$ ge dieser Veranstaltung - sie dient gleichsam als Evaluierung der bisherigen Aktivität des Netzes - von fünf ZHNGruppen Poster zur Entstehungsgeschichte der einzelnen ZHN-Gruppen, drei Poster zu gruppenspezifischen inhaltlichen Schwerpunktsetzungen (Aktivitäten) und sieben Poster, die die Erfolgslinien der jeweiligen ZHN-Gruppe (einschließlich der Stolpersteine) visualisieren, erstellt. Diese Poster wurden von den Autoren inhaltsanalytisch ausgewertet. Fokussiert wurde dabei auf die inhaltliche Ebene der Poster, die gruppenspezifische Aktivität, die seitens der ZHN-Gruppen entlang einer Zeitleiste eingetragenen Erfolge bzw. Misserfolge sowie die Meilensteine und Hindernisse. Die Inhalte der Poster wurden in einen Text übertragen, der themenzentriert und gruppenvergleichend ausgewertet wurde. Da die auf den Postern verwendeten Begriffe zur Bezeichnung derselben Gegenstände kaum voneinander abweichen, wurde auf das Kodieren von Textpassagen verzichtet und gleich zur Häufigkeitsauszählung bestimmter Textmerkmale (vgl. Merten/Teipen 1991: 103) übergegangen.

\section{Ergebnisse}

Die im Folgenden vorgestellten Ergebnisse entstanden durch das Zusammenführen der quantitativen und qualitativen empirischen Befunde.

\subsection{Mitgliederstruktur und demographisches Profil der engagierten Personen}

Seit 2011 haben sich 348 Personen im Zeit-Hilfs-Netz Steiermark registrieren lassen. Am 31. Dezember 2014 hatte das Zeit-Hilfs-Netz Steiermark 229, ein Jahr später 252 eingetragene Mitglieder, obwohl zwischenzeitlich zwei Gruppen ihre Tätigkeit eingestellt haben. Die zum 31. Dezember 2015 höhere Mitgliederzahl ist auf die steigenden Mitgliederzahlen in sieben von acht ZHN-Gruppen sowie auf die kontinuierliche Teilhabe der bereits in den ZHN-Gruppen registrierten Mitglieder zurückzuführen. Nach Ansicht der
Gruppenleiter begründet sich das Ausscheiden einzelner Mitglieder hauptsächlich aus Wohnstandortwechseln bzw. aus deren Ableben. Etwas mehr als die Hälfte der sich aktuell engagierenden Personen war bereits im Jahr davor aktiv, und die Anzahl der registrierten Mitglieder, die mehr als eine Leistung anbieten bzw. nachfragen, ist niedriger als noch vor einem Jahr (vgl. Tabelle 2).

Hinsichtlich der Anzahl an aktiven Mitgliedern und der Spannweiten der aktiven Mitglieder unterscheiden sich die ZHN-Gruppen deutlich voneinander: Die Kleinstadt Trofaiach verzeichnet 44 aktiv anbietende und 31 nachfragende Personen. In den anderen sieben derzeit aktiven $\mathrm{ZHN}$ Gruppen sind zwei bis 25 anbietende Personen bzw. eine bis sieben nachfragende Personen verzeichnet.

Nach Ansicht der Gruppenleiter lassen sich die Veränderungen der Mitgliederzahlen ebenso wie die Auflösung von ZHN-Gruppen aus den Herausforderungen der Mitgliederwerbung, den unterschiedlichen (individuellen) Möglichkeiten der Aufrechterhaltung des Engagements und der vergleichsweise geringen Nachfrage nach angebotenen Leistungen erklären. Als zentrale Herausforderungen der Mitgliederwerbung werden Schwellenangst, das Nicht-annehmen-Können von Hilfe durch familienferne Personen, die Ansicht, (als älterer Mensch) keine Gegenleistung anbieten zu können, sowie die Sorge, dass die Mitgliedschaft ${ }^{12}$ im Zeit-Hilfs-Netz einen parteipolitischen Zusammenhang begründet, genannt. Die im Vergleich zum Angebot geringe Nachfrage wird auf die nicht erfüllbaren überzogenen Erwartungen der (ehemaligen) Nachfragenden in Bezug auf die Regelmäßigkeit der Unterstützung und die enttäuschte Hoffnung auf Substitution kostenintensiver professioneller (handwerklicher) Dienste zurückgeführt. Übrig bleiben somit jene (potenziellen) Nachfrager, die sich des Erfordernisses von (gelegentlicher) Unterstützung bewusst sind, um Hilfe bitten können und der eigenen Einsamkeit aktiv etwas entgegensetzen möchten. Soziale Motive stehen für sie, wie Blüml (2015: 87 f.) für eine ZHN-Gruppe festgestellt hat, im Vordergrund.

Die Voraussetzung und Motivation zur Teilhabe am ZeitHilfs-Netz Steiermark besteht nach Ansicht der Gruppenleiter darin, etwas Sinnvolles tun zu wollen, rasch und unbürokratisch unterstützungssuchenden Personen helfen zu können sowie die Möglichkeit zu haben, die hierfür notwendigen Zeitreserven zu mobilisieren und die Unterstützungsaktionen in den eigenen Alltag gut integrieren zu können. Unlängst zugezogene Personen sehen in diesem Zusammenhang eine gute Gelegenheit, sich in der neuen Wohngemeinde rascher zu integrieren. Personen, die mit plötz-

\footnotetext{
12 Unter Mitgliedschaft wird hier die formelle Zugehörigkeit zu einem Verein verstanden, die grundsätzlich per Vertrag begründet wird. Die daraus entstehenden Pflichten und Rechte sind in den jeweiligen Vereinsstatuten geregelt (vgl. Österreichisches Vereinsgesetz 2002).
} 
Tabelle 2 Eckdaten zu den im Zeit-Hilfs-Netz Steiermark registrierten Mitgliedern

\begin{tabular}{llll}
\hline & 31. Dezember 2014 & 31 . Dezember 2015 & $\begin{array}{l}\text { zu beiden Zeitpunk- } \\
\text { ten }\end{array}$ \\
aktive ZHN-Gruppen & 8 & 8 & 6 \\
Mitglieder & 229 & 252 & 133 \\
unter 20-Jährige & $7(3 \%)$ & $8(3 \%)$ & $7(5 \%)$ \\
60-Jährige und Ältere & $86(38 \%)$ & $109(43 \%)$ & $53(40 \%)$ \\
Relation Frauen : Männer & $3: 1$ & $2: 1$ & $2: 1$ \\
aktive Mitglieder & 133 & 119 & 49 \\
unter 20-Jährige & $4(3 \%)$ & $8(7 \%)$ & $4(8 \%)$ \\
60-Jährige und Ältere & $47(35 \%)$ & $42(35 \%)$ & $15(31 \%)$ \\
Relation Frauen : Männer & $3: 1$ & $2: 1$ & $3: 1$ \\
Anbieter & 107 & 96 & 44 \\
darunter Mehrfachanbieter & 59 & 51 & 19 \\
unter 20-Jährige & $4(4 \%)$ & $8(8 \%)$ & $4(9 \%)$ \\
60-Jährige und Ältere & $33(31 \%)$ & $26(27 \%)$ & $13(30 \%)$ \\
Relation Frauen : Männer & $3: 1$ & $2: 1$ & $3: 1$ \\
Nachfrager & 58 & 46 & 12 \\
darunter Mehrfachanbieter & 17 & 8 & 1 \\
unter 20-Jährige & $1(2 \%)$ & $2(4 \%)$ & $1(8 \%)$ \\
60-Jährige und Ältere & $23(40 \%)$ & $26(57 \%)$ & $4(33 \%)$ \\
Relation Frauen : Männer & $4: 1$ & $5: 1$ & $5: 1$ \\
Anbieter und Nachfrager & 32 & 23 & 7 \\
unter 20-Jährige & $1(3 \%)$ & $2(9 \%)$ & $1(14 \%)$ \\
60-Jährige und Ältere & $9(28 \%)$ & $10(43 \%)$ & $2(29 \%)$ \\
Relation Frauen : Männer & $4: 1$ & $5: 1$ & $6: 1$ \\
\hline
\end{tabular}

lich veränderten Lebensumständen konfrontiert sind, freuen sich auf neue Kontakte. Die Anbieter wiederum erwarten keine Gegenleistung. Ihnen genügt es, wenn ihre Angebote nachgefragt werden. Andernfalls werden sie ungeduldig und scheiden aus dem Zeit-Hilfs-Netz Steiermark aus.

Die Beteiligung von Frauen liegt zu beiden Zeitpunkten über jener der Männer, auch bei den Mehrfachanbietenden (vgl. Tabelle 2). Das Verhältnis von Frauen zu Männern, die zu beiden Zeitpunkten mehrere Hilfestellungen anbieten, beträgt rund 3:1. Die Analyse der nachfragenden Mitglieder zeigt Ähnliches: Zu beiden Zeitpunkten besteht ein Frauenüberhang. Das Verhältnis Frauen zu Männer, die zu beiden Zeitpunkten anbieten, liegt bei 5:1. Die Gruppenleiter vermuten hinter der überproportionalen Teilhabe von Frauen ein ausgeprägteres Bewusstsein für soziale Anliegen, mehr Kommunikationsfreude und Interesse an persönlichem Austausch, hinter der geringeren Beteiligung von Männern das Engagement in anderen Vereinen und Organisationen und ein höheres Maß an Überwindung, sozial tätig zu werden.

$\mathrm{Zu}$ beiden Zeitpunkten stellen Personen im Erwerbsalter (20 bis 59 Jahre) gefolgt von den über 60-Jährigen den größten Anteil unter den (aktiven) Mitgliedern, während Personen über 60 Jahre am häufigsten nachfragen. Die Altersstruktur der aktiven Mitglieder ist in den ZHN-Gruppen unterschiedlich: Teilweise fehlen ganze Alterskohorten (vor allem die 20- bis 39-Jährigen), eine einzige ZHN-Gruppe ist altersstrukturell ausgewogen. Dies führen die Mitarbeiter der Landentwicklung Steiermark auf die intensive Bewerbung und Betreuung der ZHN-Gruppe in der und durch die Standortgemeinde zurück.

Junge Menschen sind mit Ausnahme einer ZHN-Gruppe, in welcher das aktive Engagement „,belohnt“ wird, ${ }^{13}$ im Zeit-Hilfs-Netz Steiermark unterrepräsentiert. Die Beteiligung der unter 20-Jährigen ist zu beiden Zeitpunkten gering: Per 31. Dezember 2014 boten vier Personen Hilfe an, eine Person fragte nach. Ein Jahr später gab es acht anbietende und zwei nachfragende junge Menschen. Diese geringe Beteiligung erklären die Gruppenleiter und die Mitarbeiter der Landentwicklung Steiermark mit der selektiven Abwanderung und der Dynamik dieser Lebensphase, die durch andere Prioritäten, knappe Zeitbudgets und geringe Präsenz vor Ort gekennzeichnet ist.

Zusammenfassend ist zu sagen, dass Frauen (74\% aller Anbietenden) (vgl. Abbildung 2) und Personen, die im Erwerbsalter sind bzw. am Übergang in die nachberufliche Lebensphase stehen (50\% aller Anbietenden), gleichsam das Rückgrat der Gebenden bilden. Dies bezieht sich einerseits auf die Beteiligung der 50-bis 69-Jährigen, an-

\footnotetext{
${ }^{13}$ Es wird ein Boni-System praktiziert, welches den Tausch von Zeit gegen freien Eintritt in kommunale Freizeiteinrichtungen ermöglicht.
} 
Abbildung 2 Anbieter nach Geschlecht (31. Dezember 2015)

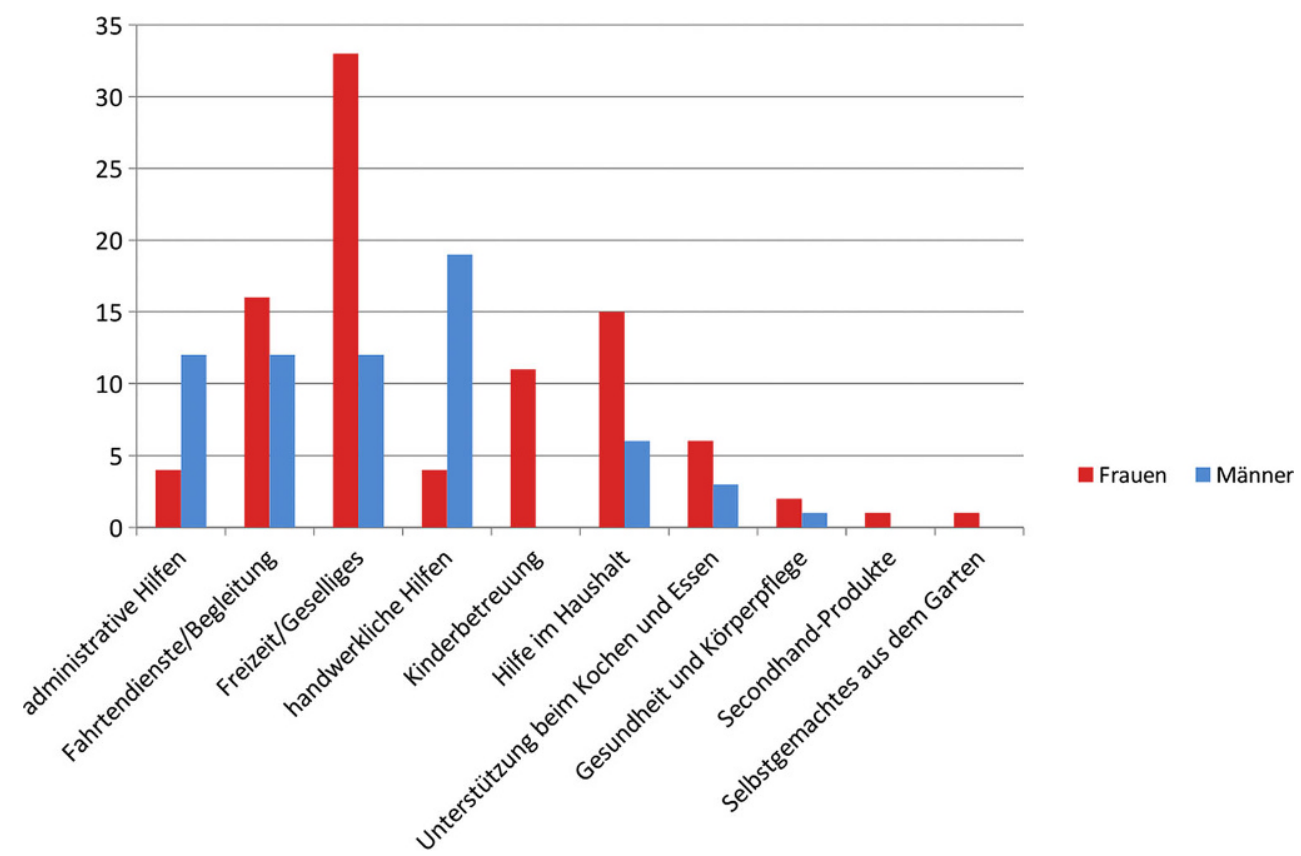

dererseits auf die thematische Bandbreite der von ihnen angebotenen (Dienst-)Leistungen.

\subsection{Qualität und Quantität der angebotenen und nachgefragten Leistungen}

\subsubsection{Vielfältiges Leistungsspektrum}

Zum Zeitpunkt der Datenanalyse wurden Leistungen in zehn Kategorien (vgl. Kapitel 5.2) angeboten bzw. nachgefragt. Die Analyse der Detailbeschreibungen der Leistungen spiegelt die inhaltliche Vielfalt und die Spezifität der Offerte wider: In der Kategorie ,administrative Hilfen“ finden sich neben allgemeinen, nicht näher spezifizierten Hilfestellungen auch Angebote zur Unterstützung beim Umgang mit dem Computer, zur Übernahme von Behördenwegen und Unterstützung bei Schulaufgaben. Die Kategorie „Fahrtendienste/Begleitung“ umfasst - abgesehen von allgemeinen, nicht näher spezifizierten Hilfestellungen - auch Angebote und Nachfragen für Einkaufsfahrten, Botengänge sowie die Begleitung zum Arzt oder zur Apotheke. Die Kategorie „Freizeit/Geselliges“ ist am stärksten ausdifferenziert und enthält allgemein gehaltene Inserate zu gemeinsamen oder begleiteten Außer-Haus-Aktivitäten (darunter vor allem Begleitung beim Spazierengehen, Hund ausführen, mit den Kindern spazieren gehen), aber auch spezifischere Angebote und Nachfragen in der häuslichen Freizeitgestaltung (wie z. B. Vorlesen, Kartenspielen, Plaudern, Stricken beibringen, Unterricht in einem bestimmten Musikinstrument). Personen, die in der Kategorie ,handwerkliche Hilfen“ Inserate schalten, suchen bzw. bieten Unterstützung vor allem bei der Übernahme von (kleineren) Reparaturarbeiten im Haus sowie bei der Gartenpflege. Die Kategorie „Hilfe beim Kochen und Essen“" umfasst Angebote für die (fallweise) Unterstützung beim Backen bzw. beim Zubereiten von Mahlzeiten. Die Angebote und Nachfragen in der Kategorie „Hilfe im Haushalt“ sind unterschiedlich detailliert beschrieben. Alle Leistungen beziehen sich auf klassische Arbeiten im Haushalt wie Fensterputzen, Bügeln oder die Betreuung der Wohnung während des Urlaubs. Leistungen im Bereich „Kinderbetreuung“ fokussieren die stundenweise Betreuung bzw. Unterstützung der Kinder in schulischen Angelegenheiten. Einzelangebote im Bereich „Gesundheit und Körperpflege" spannen den Bogen von der Gesprächstherapie bis hin zur Hospizbegleitung. In den beiden Kategorien „Selbstgemachtes aus dem Garten“ und „Secondhandprodukte" finden sich vereinzelt Angebote, die nicht näher spezifiziert sind.

\subsubsection{Kategorienbezogene Schwerpunkte und die Relation von Angebot und Nachfrage}

Am häufigsten sind Angebote in den Kategorien „Freizeit/ Geselliges“, „Fahrtendienste/Begleitung“, „handwerkliche Hilfen“ und „Hilfe im Haushalt". Sie stammen von Frauen im Alter von 50 bis 69 Jahren. Männer engagieren sich vor allem in den Bereichen ,handwerkliche Hilfen“ und „,administrative Hilfen“, bieten Fahrtendienste, Begleitungen sowie Unternehmungen im Rahmen der gemeinsamen Freizeitgestaltung an. Ein ausgedünntes Angebot gibt es in den Kategorien „Gesundheit und Körperpflege“ sowie „Kinderbetreuung“. Mit Ausnahme der Kategorie „Freizeit/ Geselliges“ zeigt die Analyse der Angebotsinserate kategorienbezogene Schwankungen. Die Aktivität der Zugehöri- 


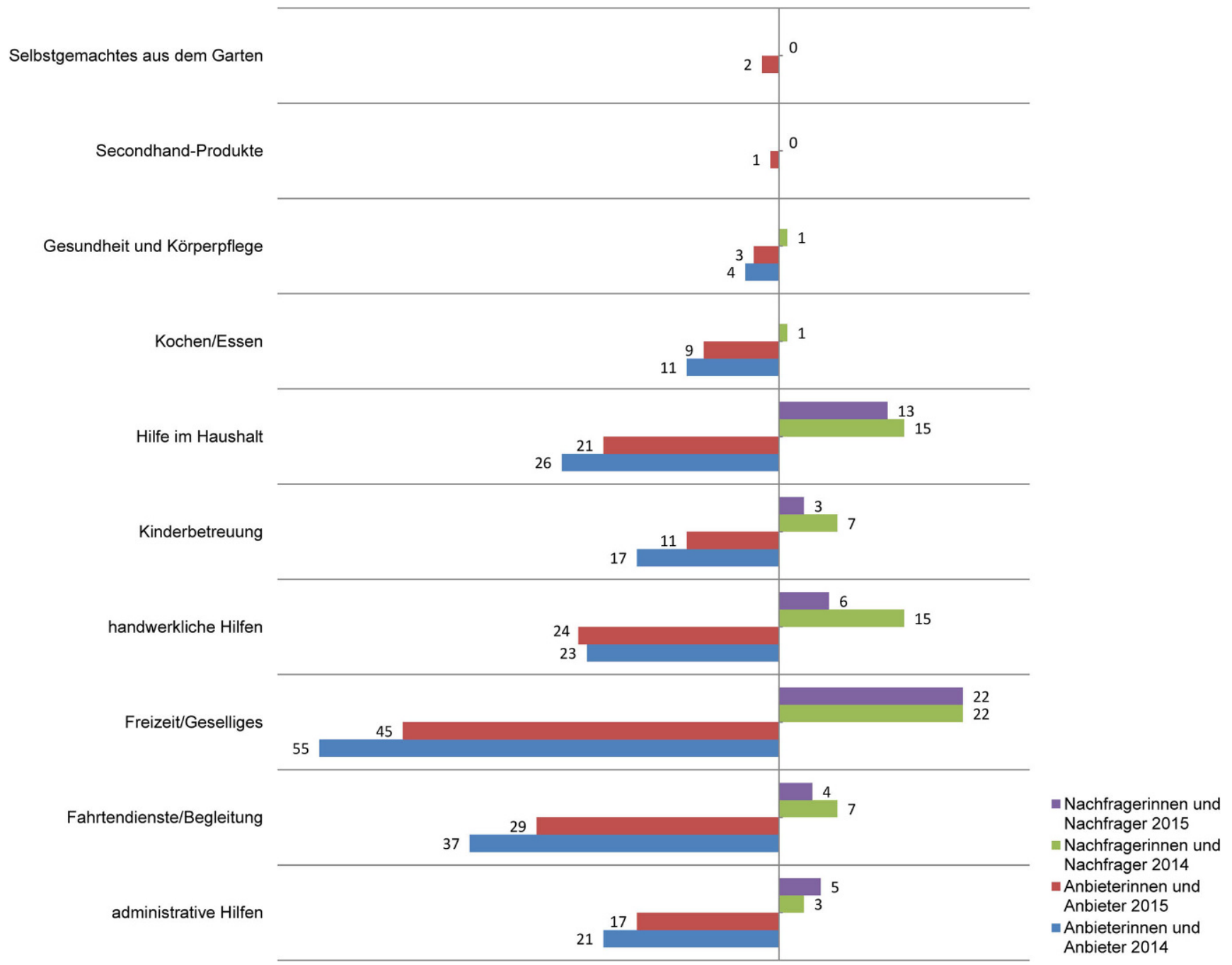

Abbildung 3 Kategorienbezogenes Angebot und Nachfrage im Vergleich

gen zu den anderen Altersgruppen hingegen streut über das Leistungsspektrum.

Die größte Nachfrage gibt es zu beiden Zeitpunkten in den Kategorien „Freizeit/Geselliges“, „Hilfe im Haushalt“ und „handwerkliche Hilfen“. In Bezug auf die Kategorie „Freizeit/Geselliges“ fällt die Bedeutung der Nachfrage nach Besuchen zuhause, Gesprächen und dem gemeinsamen Spazierengehen auf. Vergleichsweise mäßig nimmt sich die Nachfrage nach Fahrtendiensten und Begleitungen aus.

Dass Personen gleichzeitig Anbieter und Nachfrager sind, stellt nicht den Regelfall, sondern die Ausnahme dar. Somit verwundert nicht, dass quantitativ das Angebot die Nachfrage überwiegt. Zum 31. Dezember 2014 boten 107 Personen 194 Hilfestellungen an, zum 31. Dezember 2015 waren es 96 Personen mit 162 Angeboten. Zu beiden Zeitpunkten werden dreimal so viele Leistungen angeboten wie nachgefragt (vgl. Abbildung 3).
Die Nachfrage kommt tendenziell von Frauen. Sie fragen vor allem in den Kategorien „Freizeit/Geselliges“ und „Hilfe im Haushalt“ nach (vgl. Abbildung 4). Hinsichtlich der altersspezifischen Nachfrage überrascht beispielsweise die der 70- bis 79-Jährigen nach Fahrtendiensten wenig. Umso erstaunlicher ist - trotz der kleinen Fallzahlen - die große Nachfrage in den Kategorien „Freizeit/Geselliges“, „handwerkliche Hilfen“ und „Hilfe im Haushalt“ über alle Altersgruppen.

Sowohl das aktuelle gruppenspezifische Angebot als auch die Nachfrage orientieren sich laut Auskunft der Gruppenleiter am momentanen Bedarf. Somit ergeben sich neben thematischen Jahresschwerpunkten - 2014 Betreuung älterer Menschen, 2015 Betreuung von Asylbewerbern - und gemeinsam festgelegten Aktivitäten (z. B. EDVKurs für Senioren in Zusammenarbeit mit Schulen, Thementage, Flohmärkte/Sammeln für gute Zwecke) auch saisonale Schwerpunkte (z. B. Tausch von Gartenpflanzen, Gartenbetreuung während des Sommerurlaubs). 
Abbildung 4 Nachfrager nach Geschlecht (31. Dezember 2015)

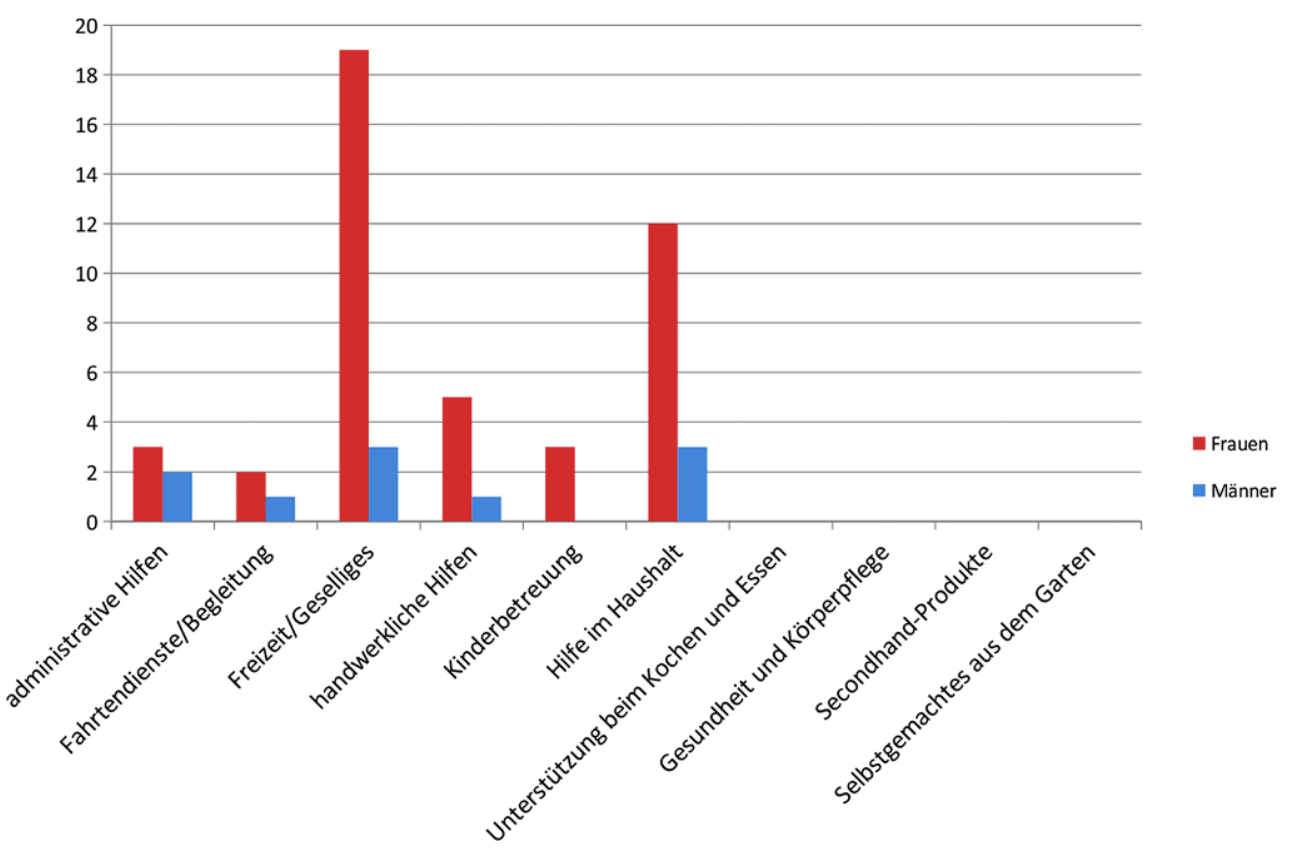

Hinsichtlich der Angebots- und Nachfragestruktur ähneln die ZHN-Gruppen einander: In jeder ZHN-Gruppe gibt es Personen, die handwerkliche Hilfen anbieten; Freizeit/ Geselliges wird mit einer Ausnahme in allen derzeit aktiven ZHN- Gruppen nachgefragt, Fahrtendienste/Begleitung werden mit Ausnahme von einer ZHN-Gruppe nicht nachgefragt, und es besteht kein Bedarf nach Unterstützung beim Kochen und Essen, bei Gesundheit und Körperpflege sowie den beiden 2015 neu eingeführten Kategorien „Secondhandprodukte“ und „Selbstgemachtes aus dem Garten". Kinderbetreuung wird ebenfalls kaum nachgefragt. Die Vermutung liegt nahe, dass es sich hierbei um einen sensiblen Bereich handelt (Verantwortung, Haftungsfragen) oder andere Betreuungsmöglichkeiten genutzt werden. Das vorhandene Angebot im öffentlichen Personennahverkehr, die (nach wie vor gegebene) eigene Pkw-Fahrtüchtigkeit sowie verlässliche informell organisierte Fahrgemeinschaften wiederum könnten die generell geringe Nachfrage nach Fahrtendiensten und Begleitung erklären.

Weshalb sich im Vergleich der beiden Zeitpunkte kategorienbezogene gegenläufige Entwicklungen von Angebot und Nachfrage in den Bereichen Freizeitgestaltung und administrative Unterstützung zeigen, lässt sich nicht hinreichend erklären. Es scheint, dass Menge und Art der über das Zeit-Hilfs-Netz Steiermark angebotenen und nachgefragten Leistungen von der Anzahl und Fluktuation der momentan aktiven Mitglieder, deren spezifischen Fertigkeiten und persönlichen Interessen sowie den spontan entstehenden Bedarfen bei sich plötzlich verändernden Lebenssituationen (z. B. Tod des Ehepartners) gesteuert werden und Trends erst durch eine Längsschnittbetrachtung sichtbar gemacht werden können.

\subsubsection{Bedingungen des Angebots und Besonderheiten der Nachfrage}

Es ist anzunehmen, dass Tauschbeziehungen dann eingegangen werden, wenn sowohl inhaltliche Übereinstimmung als auch Einvernehmen über die Zeitlichkeit und Räumlichkeit der Leistungserbringung gegeben und die Erreichbarkeit gewährleistet sind. Angesichts dieser Erfordernisse bringt die Analyse der Inserate Interessantes ans Licht: Es gibt einen Überhang an unregelmäßigen Angeboten, während die Nachfragenden zu beiden Zeitpunkten den Wunsch nach regelmäßiger Unterstützung signalisieren (vgl. Abbildung 5).

Hinter der Unregelmäßigkeit der Angebote ist die fehlende Bereitschaft der Anbietenden zur zeitlichen Bindung oder der Wunsch nach Spontaneität in der Hilfestellung (vgl. Carroll 2013: 140; Shih/Bellotti/Han et al. 2015: 1081) zu vermuten. Die Bedeutung anderer Einflussfaktoren kann auf der Basis der vorliegenden empirischen Befunde nicht abgeleitet werden.

Im Vergleich der beiden Zeitpunkte fallen neben dem quantitativen Rückgang der Nachfrage auch kategorienspezifische Verschiebungen hin zur Nachfrage nach Unterstützung im Bedarfsfall auf. Während ein Jahr zuvor die Nachfrager einen tendenziell regelmäßigen Bedarf in den Kategorien „Fahrtendienste/Begleitung“, „Freizeit/Geselliges“, „,handwerkliche Hilfen“, „Kinderbetreuung“ und „Hilfe im Haushalt" anmeldeten, ist die Nachfrage zum 31. Dezember 2015 - mit Ausnahme der Kategorien „Fahrtendienste/ Begleitung“ und „Kinderbetreuung“ - tendenziell anlassfallbezogen. Die Gruppenleiter erklären diese Entwicklung mit der ihrerseits geleisteten Aufklärungsarbeit zwecks Er- 

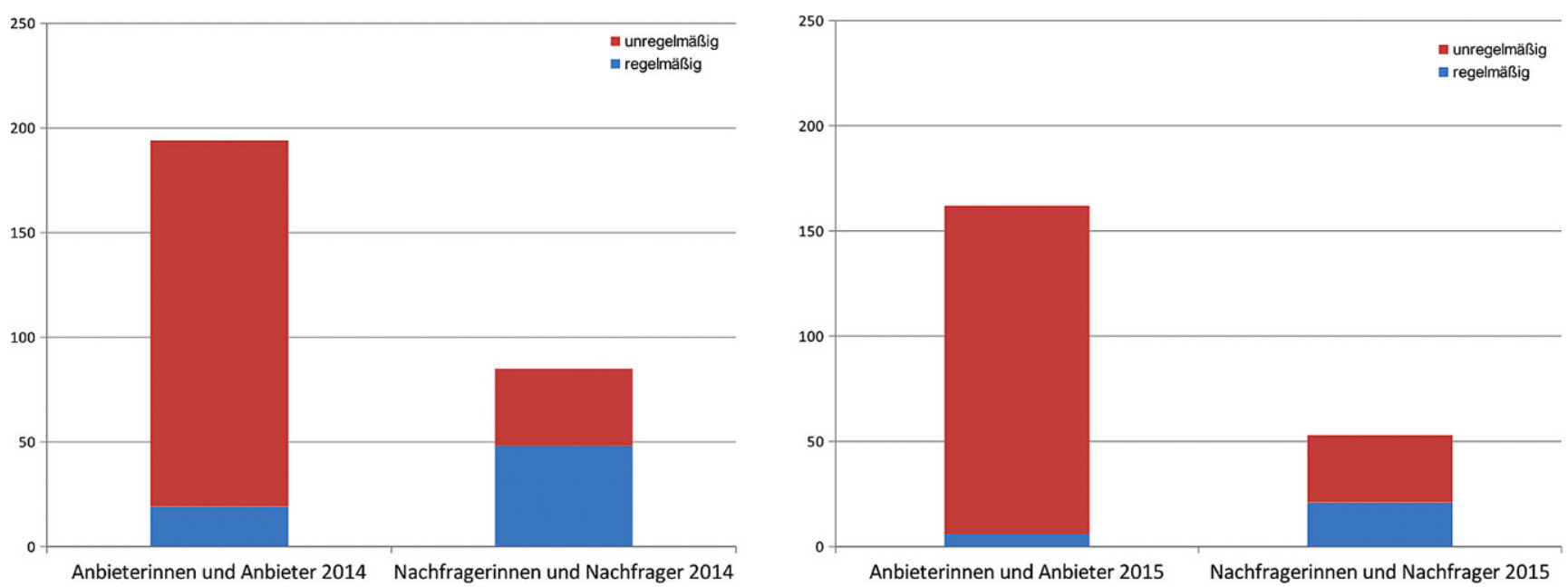

Abbildung 5 Anbieter- und nachfragerspezifische Vorstellungen über die Zeitlichkeit des Tausche(n)s

dung der Ansprüche und Erwartungen der Nachfragenden ${ }^{14}$ durch den permanenten Hinweis auf die Freiwilligkeit der im Zeit-Hilfs-Netz Steiermark erbrachten Leistungen und die scharfe Abgrenzung zu professionellen Diensten.

Die Analyse der 141 Angaben für den ersten und der 120 Angaben für den zweiten Zeitpunkt zu den räumlichen Reichweiten bzw. seitens der Anbietenden erforderlichen Aktionsradien zeigt, dass das häusliche Umfeld sowie die (eigene) Wohngemeinde die beiden wichtigsten räumlichen Bezugsebenen der Leistungserbringung und -nachfrage sind (vgl. Abbildung 6).

Zudem werden die kategorienspezifisch unterschiedlichen Mobilitätserfordernisse der Anbietenden sichtbar: Sowohl bei den administrativen Hilfen als auch bei „Freizeit/ Geselliges" spielt die Wohngemeinde des Anbieters und des Nachfragers eine zentrale Rolle. ${ }^{15}$ Administrative Hilfen und Beiträge im Bereich „Freizeit/Geselliges“ werden vornehmlich für Personen, die in derselben Wohngemeinde wie die Anbieter wohnen, geleistet. In Bezug auf „Fahrtendienste/Begleitung" geht der Aktionsradius der Anbietenden über die eigene Wohngemeinde hinaus und richtet sich (theoretisch) (partiell) nach den Ansprüchen der Nachfragenden. Diesen wiederum ist es tendenziell ein Anliegen, beispielsweise zwecks gemeinsamer Freizeitgestaltung (vor allem Spazierengehen, Kartenspielen) oder für administrative Hilfen die Wohngemeinde nicht verlassen zu müssen. In Bezug auf die Kategorie „Fahrtendienste/Begleitung“ beschreiben die Nachfragenden die Zielorte nicht näher, son-

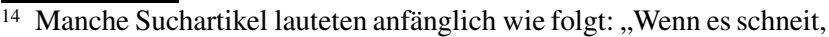
möchte ich, dass mein Gehsteig um 6 Uhr morgens gereinigt wird.“

15 Explizit keine Angaben zum Ort der Leistungserfüllung machen die Anbieter und Nachfrager bei den Detailbeschreibungen der Kategorien „Kinderbetreuung“, „handwerkliche Hilfen“ und „Hilfe im Haushalt“. Dies lässt die Schlussfolgerung zu, dass diese Leistungen zuhause bei den Nachfragenden erbracht werden.
}

dern erläutern den mit der Fahrt verbundenen Zweck und bekunden die Absicht, selbst mitzufahren.

\subsection{Zur Übereinstimmung von Angebot und Nachfrage und zur Bedeutung des Tauschens}

Die inhaltliche, zeitliche und aktionsräumliche Übereinstimmung von Angebot und Nachfrage bildet die Voraussetzung für eine (theoretische) Tauschbeziehung. Die Analyse der Inserate (vgl. Tabelle 3) ergibt, dass sich aus den aktuell 106 registrierten Anbietern, 52 registrierten Nachfragern und 131 aktiven Mitgliedern theoretisch 160 Tandems bilden lassen, von denen insgesamt 69 Personen profitieren (könnten).

Die Entfernungen zwischen den Wohnstandorten der (theoretisch) zusammenpassenden Anbietenden und Nachfragenden reichen von 78 bis einigen Hundert Metern bis hin zu mehr als 12 Kilometern in Kleinstädten wie auch Landgemeinden. Der zeitliche Aufwand für die Distanzüberwindung beträgt in den Standortgemeinden tendenziell mehr als fünf Autominuten. Die geringen Fallzahlen und die Zufallsbedingtheit der theoretischen Passung lassen keine raumtypenspezifischen Schlussfolgerungen für die zeitlichen Aufwände für die Distanzüberwindung der Tauschpartner zu. Für das Zustandekommen von Tauschbeziehungen scheint in Anbetracht der Altersstruktur der Helfenden die Pkw-Fahrtüchtigkeit Voraussetzung für das aktive Anbieten zu sein (vgl. auch Blüml 2015: 90), zumal in den ländlichen Fallbeispielgemeinden die innergemeindliche Erschließung mittels öffentlichem Personennahverkehr meist nicht gegeben oder dessen Nutzung im Vergleich zum Pkw zu aufwändig ist. Die Bequemlichkeit durch das Auto ist vor allem dann von Belang, wenn ein Anbieter mehrere Nachfragende bedient und es in dispersen Sied- 
Anbieterinnen und Anbieter 2014

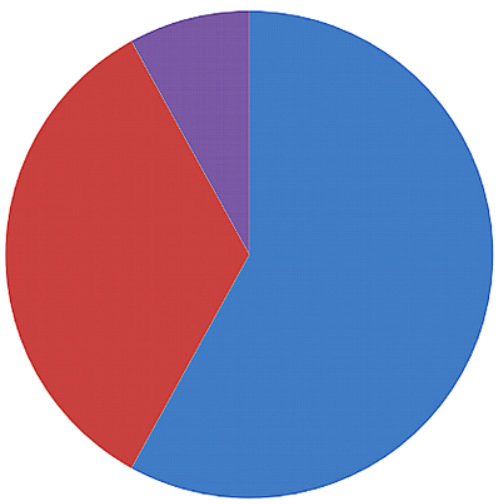

a innerhalb der Wohngemeinde Wohngemeinde

$\square$ außerhalb der Wohngemeinde aktionsradius unbestimmt - inner- und außerhalb der

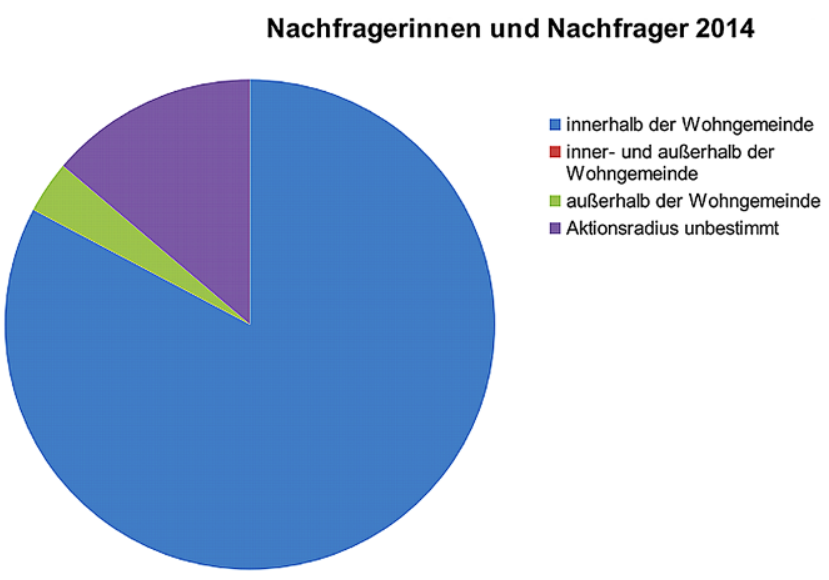

Anbieterinnen und Anbieter 2015

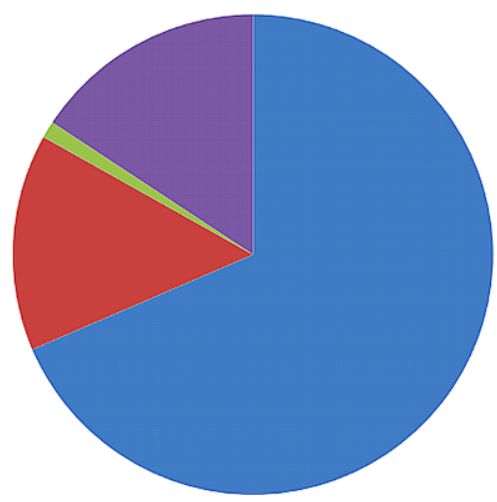

G innerhalb der Wohngemeinde a inner- und außerhalb der Wohngemeinde

a außerhalb der Wohngemeinde aktionsradius unbestimmt

Abbildung 6 Erforderliche Reichweiten der angebotenen und nachgefragten Leistungen

lungsstrukturen mit bewegter Topographie weite Wege zurücklegen muss.

Die Reziprozität, das heißt das wechselseitige Geben und Nehmen, lässt sich auf Basis der zur Auswertung vorliegenden Informationen nicht bestimmen. Die Ergebnisse des Ergebnisprotokolls bestätigen die Einschätzung der Gruppenleiter in Bezug auf die Bedeutung des Tauschprinzips: Das gleichzeitige Anbieten und Nachfragen von Unterstützung ist in allen ZHN-Gruppen die Ausnahme. Selbst die intensive Bewerbung des Zeit-Hilfs-Netzes Steiermark und die Aufklärungsarbeit seitens der Gruppenleiter ändern daran nichts. Trotzdem sei der Bestand des Zeit-Hilfs-Netzes Steiermark nach Ansicht der Gruppenleiter nicht gefährdet. Es habe sich lediglich der Wandel des Zeit-HilfsNetzes Steiermark weg von den Tauschaktionen hin zu einer Plattform der Begegnung, wo das Zusammenkommen, Freundschaft schließen und die gemeinsame Betätigung der Mitglieder im Vordergrund stehen, vollzogen. Vielmehr sei es als Erfolg zu werten, dass aus dem Zeit-Hilfs-Netz Steiermark ausgeschiedene Personen einander weiterhin treffen und sich informell unterstützen. Man solle sich darüber freuen, dass die Nachbarschaftshilfe wieder funktioniert.
Hinsichtlich der quantitativen Dimension kommt dem Zeit-Hilfs-Netz Steiermark nach Ansicht der Gruppenleiter in der Daseinsvorsorge flankierende Bedeutung $\mathrm{zu}$. Auch sei das Funktionieren des Zeit-Hilfs-Netzes durch den Rückbau der kommunalen Basisinfrastruktur weder bedroht, noch sei zu befürchten, dass das Netz diese irgendwann gar ersetzen solle. Auch müsse im Hinblick auf die Zukunft des Zeit-Hilfs-Netzes die Bedeutung von (statistischen) Kennzahlen zur Evaluierung von erfolgreich organisierter Nachbarschaftshilfe reflektiert und diskutiert werden, um nicht in die Rechtfertigungsfalle zu tappen.

\section{Diskussion der empirischen Befunde}

Da es sich bei den empirischen Ergebnissen zum Zeit-HilfsNetz Steiermark um Momentaufnahmen handelt, ist Behutsamkeit bei der Ableitung des Mehrwerts dieser Zeitbank für die Lebensqualität und Potenziale der Beteiligten im Kontext der Daseinsvorsorge geboten. Der hohe Detaillierungsgrad der empirischen Befunde zum Zeit-Hilfs-Netz Steiermark lässt eine inhaltlich-strukturelle Einordnung in Bezug auf andere österreichische Zeitbanken nicht $\mathrm{zu}$, wohl 
Tabelle 3 Die Passfähigkeit von Angebot und Nachfrage

\begin{tabular}{|c|c|c|c|c|}
\hline $\begin{array}{l}\text { ZHN-Gruppe } \\
\text { (aktuellster Zeit- } \\
\text { punkt) }\end{array}$ & $\begin{array}{l}\text { registrierte } \\
\text { Anbieter }\end{array}$ & $\begin{array}{l}\text { registrierte } \\
\text { Nachfrager }\end{array}$ & $\begin{array}{l}\text { aktive } \\
\text { Mitglieder }\end{array}$ & theoretische Passungen (Tandems) \\
\hline Bad Radkersburg & 0 & 0 & 0 & 0 \\
\hline Bärnbach & 13 & 7 & 14 & $\begin{array}{l}\text { Freizeit/Geselliges: } \\
\text { Tandems: 1, involviert: } 2 \text { Personen } \\
750 \mathrm{~m}: 3 \text { Autominuten oder } 9 \text { Minuten Gehzeit } \\
\text { handwerkliche Hilfen: } \\
\text { Tandems: } 2 \text {, involviert: } 4 \text { Personen (darunter } 2 \text { Nachfragende) } \\
150 \mathrm{~m} \text { bzw. } 900 \mathrm{~m}: 1 \text { Autominute oder } 2 \text { bzw. } 11 \text { Gehminuten } \\
\text { Hilfe im Haushalt: } \\
\text { Tandems: } 7 \text {, involviert: } 6 \text { Personen (darunter } 4 \text { Nachfragende) } \\
850 \mathrm{~m}-3,5 \mathrm{~km}: 4 \text { bzw. } 7-8 \text { Autominuten oder } 10 \text { bzw. } 40 \text { Gehminuten }\end{array}$ \\
\hline Deutschfeistritz & 6 & 3 & 6 & $\begin{array}{l}\text { administrative Hilfen: } \\
\text { Tandems: } 2 \text {, involviert: } 3 \text { Personen (darunter } 2 \text { Nachfragende) } \\
8,7 \text { bzw. } 9,4 \mathrm{~km}: 12-13 \text { Autominuten }\end{array}$ \\
\hline Eibiswald & 0 & 0 & 0 & 0 \\
\hline Gleisdorf & 9 & 6 & 11 & $\begin{array}{l}\text { Fahrtendienste/Begleitung: } \\
\text { Tandems: 1, involviert: } 2 \text { Personen } \\
6,2 \mathrm{~km}: 7 \text { Autominuten } \\
\text { handwerkliche Hilfen: } \\
\text { Tandems: } 1 \text {, involviert: } 2 \text { Personen } \\
\text { 3,4 km: } 5 \text { Autominuten }\end{array}$ \\
\hline Gratwein-Straßengel & 2 & 2 & 2 & 0 \\
\hline Graz-Südost & 6 & 1 & 6 & 0 \\
\hline Zonta Feldbach & 1 & 0 & 1 & 0 \\
\hline $\begin{array}{l}\text { St. Georgen an der } \\
\text { Stiefing }\end{array}$ & 25 & 2 & 25 & $\begin{array}{l}\text { handwerkliche Hilfen: } \\
\text { Tandems: } 1 \text {, involviert: } 2 \text { Personen } \\
65 \text { m: } 1 \text { Minute Gehzeit }\end{array}$ \\
\hline Trofaiach & 44 & 31 & 66 & $\begin{array}{l}\text { Fahrtendienste/Begleitung: } \\
\text { Tandems: } 27 \text {, involviert: } 11 \text { Personen (darunter } 3 \text { Nachfragende) } \\
800 \mathrm{~m}-4,1 \mathrm{~km}: 2-10 \text { Autominuten bzw. für } 800 \mathrm{~m} 10 \text { Gehminuten } \\
\text { Freizeit/Geselliges: } \\
\text { Tandems: } 100 \text {, involviert: } 27 \text { Personen (darunter } 8 \text { Nachfragende) } \\
78 \mathrm{~m}-7,8 \mathrm{~km}: 3 \text { Personen unter } 400 \mathrm{~m} \text { und weniger als } 10 \text { Gehminuten; } \\
\text { Rest: } 1-15 \text { Autominuten } \\
\text { Hilfe im Haushalt: } \\
\text { Tandems: } 16 \text {, involviert: } 10 \text { Personen (darunter } 8 \text { Nachfragende) } \\
1,4-12,5 \mathrm{~km}: 5-11 \text { Autominuten, keine Personen unter } 10 \text { Gehminuten }\end{array}$ \\
\hline
\end{tabular}

aber einen Vergleich in Bezug auf die Mitgliederzahlen. Höllhumer und Trukeschitz (2016: 16) stellen fest, dass die durchschnittliche Mitgliederzahl auf der Organisationsebene 139 Mitglieder, auf der lokalen Ebene 32 Mitglieder umfasst. Somit liegt das Zeit-Hilfs-Netz Steiermark auf der Organisationsebene mit einer Mitgliederzahl von knapp 350 Personen weit über dem und mit 31 Personen auf lokaler Ebene (ZHN-Gruppen) im Durchschnitt.

Am Beispiel des Zeit-Hilfs-Netzes Steiermark lässt sich zeigen, dass Zeitbanken vorrangig von Frauen mittleren Alters getragen werden. Dies weicht zwar von den Ergebnissen einer österreichweiten Studie (IFES 2013) ab, die ein ausgewogenes Geschlechterverhältnis im Bereich der informellen Freiwilligenarbeit und einen Männerüberhang in der formellen Freiwilligenarbeit identifiziert, reiht sich aber in die internationalen Befunde zu Zeitbanken ein (vgl. Neymotin 2016: 155). Die geschlechtsspezifischen Beson- derheiten des Angebots - Frauen bieten bevorzugt Unterstützung in den Bereichen „Freizeit/Geselliges“ und „Hilfe im Haushalt“" an, Männer im Bereich ,handwerkliche Hilfen“ - decken sich mit der geschlechterspezifischen Struktur der informellen Freiwilligenarbeit in Österreich (IFES 2013: 40). Ob ein ausgewogeneres Geschlechterverhältnis und die Erhöhung der Beteiligung junger Menschen vorhandene Lücken im Portfolio des Zeit-Hilfs-Netzes Steiermark (z. B. Tätigkeiten, die mit körperlichen Belastungen verbunden sind) schließen könnten, bleibt ebenso unbeantwortet wie die Frage, inwiefern das Engagement in anderen Vereinen (wie beispielsweise Sport- und Musikvereinen) oder das berufliche Fortkommen junge Menschen von einer Teilhabe im Zeit-Hilfs-Netz Steiermark bzw. an organisierter Nachbarschaftshilfe abhält (Pantea 2015: $277 \mathrm{ff}$.) bzw. inwiefern die Art der im Zeit-Hilfs-Netz Steiermark 
nachgefragten Leistungen den Interessen junger Menschen entgegensteht.

Die quantitative Bedeutung der im Zeit-Hilfs-Netz Steiermark angebotenen und nachgefragten Leistungen ist trotz der kleinräumigen Organisationsstruktur gering. Das Angebot überwiegt die Nachfrage, und es dominieren Handreichungen in Bezug auf die Freizeitgestaltung, Hilfe im Haushalt und handwerkliche Unterstützung. Dieser empirische Befund bringt damit einen international belegten zentralen Stolperstein für Zeitbanken ans Licht: nicht um Hilfe bitten zu können oder zu wollen (nef 2008: 1; Valor/ Papaoikonomou 2016: 9). Die im Zeit-Hilfs-Netz Steiermark verhältnismäßig große Bedeutung von Angeboten und Nachfragen im Bereich „Freizeit/Geselliges“ für die Zugehörigen aller Altersgruppen und die kurzen Distanzen zwischen den Wohnstandorten der theoretischen Tandempartner lassen vermuten, dass die - möglicherweise auch unterschiedlichen sozialen Milieus zugehörigen - Menschen einander im Alltag bzw. im öffentlichen Raum nicht (mehr) begegnen (Dangschat/Hamedinger 2007: 228) bzw. sich nicht einfach so im Vorbeigehen über ihre Bedarfe austauschen. Abseits der Einzelbeiträge, die im Zeit-HilfsNetz im Kontext der Daseinsvorsorge erbracht werden, zeigt sich, dass durch die Teilhabe am Netz jedenfalls individuelle Mehrwerte für das subjektive Wohlbefinden ${ }^{16}$ entstehen: Die aktiv Gebenden tun etwas in deren Augen Sinnvolles und erwarten dafür Anerkennung anstelle von Gegenleistungen (vgl. auch nef 2008: 3; Shih/Bellotti/Han et al. 2015: $1078 \mathrm{ff}$.) und freuen sich über die Gelegenheit, über das Zeit-Hilfs-Netz Freundschaften zu schließen, sich mit Gleichgesinnten auszutauschen und an die Community anzudocken (vgl. Pacione 1997: 421 f.). ${ }^{17}$ Offenbar sehen sowohl die Anbietenden als auch die Nachfragenden in der Teilhabe am Zeit-Hilfs-Netz Steiermark eine Möglichkeit, der Einsamkeit zu entfliehen.

Über die Robustheit und die Bedeutung organisierter Nachbarschaftshilfe im Allgemeinen und von Zeitbanken im Speziellen im Stufenbau der Daseinsvorsorge muss angesichts der demographischen, gesellschaftlichen Verschiebungen und der Wirkkraft weiterer raumbezogener Einflussfaktoren kritischer denn je diskutiert werden, selbst wenn gesellschaftliche Mehrwerte auf ein koproduktives Moment schließen lassen.

Angesichts des hohen Anteils bereits heute älterer im Netz aktiver Personen, der geringen Anzahl der Inserate

\footnotetext{
${ }^{16}$ Subjektives Wohlbefinden wird in diesem Zusammenhang als Ergebnis von Lebensumständen interpretiert und stellt ein Konstrukt aus Lebenszufriedenheit und der Summe aller als positiv wahrgenommenen Einflussfaktoren dar (Lamu/Olsen 2016: 176).

17 Mehrwerte für die körperliche Gesundheit und die Verbesserung des Selbstwertgefühls, wie sie den Zeitbanken von internationalen Autoren (vgl. Peacock 2000: 58; nef 2008: 6; Slay 2011: 5) zugeschrieben werden, lassen sich aus den erhobenen empirischen Daten nicht ableiten.
}

und der zeitlichen und räumlichen Spezifika der angebotenen und nachgefragten Leistungen handelt es sich um einen Glücksfall, wenn eine angebotene Leistung auf eine passgenaue Nachfrage und umgekehrt trifft. Im Zeit-Hilfs-Netz Steiermark ist man sich dessen bewusst und akzeptiert, dass der Tauschgedanke nicht mehr im Vordergrund steht. Der Wandel des Netzes in Richtung gemeinschaftliche Aktivität mündet in die Frage, ob es noch dem Modell Zeitbank zuordenbar ist und wie es sich in das Angebot bestehender anderer Vereine bzw. bürgerschaftlicher Genossenschaften einordnen lässt.

An der Optimierung seiner Selbstverortung im Stufenbau des Vereinswesens bzw. gar der Daseinsvorsorge arbeitet das Zeit-Hilfs-Netz Steiermark durch die Verbesserung der Kommunikation und Koordination mit anderen Vereinen und Professionisten bereits - zwecks klarer Abgrenzung ehrenamtlich erbringbarer Leistungen von kommerziellen Angeboten - auch aus Gründen der Qualitätssicherung, Versorgungssicherheit und Haftung. Somit können aus heutiger Sicht ehrenamtlich engagierte Personen das vorhandene (kommerzielle) Angebot auf kleinräumiger bzw. kommunaler Ebene bestenfalls ergänzen, nicht aber substituieren.

In diesem Zusammenhang ist angesichts der erwartbaren altersstrukturellen Verschiebungen im Profil der aktiven Personen verstärkt eine Auseinandersetzung darüber notwendig, inwiefern anderwärtiges formelles Engagement (z. B. in Vereinen) oder informelles Engagement im Rahmen der klassischen Nachbarschaftshilfe einen Hinderungsgrund für die Beteiligung an organisierter Nachbarschaftshilfe darstellt (Baumgartner/Kolland/Wanka 2013: 151), wie sich das Angebotsportfolio verändern wird, sobald erste körperliche Einschränkungen bei den Helfenden auftreten und die Pkw-Fahrtüchtigkeit als eine der zentralen Voraussetzungen zu helfen verloren geht, die Nachfragenden mehr Unterstützung in der Alltagsorganisation benötigen und sich die Bereitschaft zur freiwilligen Übernahme von Leistungen in der Freizeit älterer wie auch jüngerer Menschen verändert (vgl. Sundeen/Raskoff 2000: 188). Die Beteiligung immobiler und sehr alter Personen wird neben der Sicherung einer kritischen Masse an sich mittelfristig aktiv Engagierenden (vgl. Shih/Bellotti/Han et al. 2015: 1081) vor allem in von Bevölkerungsrückgang geprägten ländlichen Räumen zur zentralen Herausforderung werden.

Um die Bedeutung organisierter Nachbarschaftshilfe im Kontext der Daseinsvorsorge weiterhin auf einem stabilen, wenngleich quantitativ geringen Niveau halten zu können, wird es erforderlich sein, Ehrenamt kleinräumig professio- 
neller zu organisieren ${ }^{18}$, intensiver zu begleiten und finanziell zu stützen. Dabei muss man sich vor Augen führen, dass die kollektive Alterung ganzer Siedlungsbereiche bzw. Stadtteile die Reanimation passgenauer Nachbarschaftshilfe zunehmend behindern und eine stabile kommunale Basisinfrastruktur (wie etwa Lebensmittelgeschäfte, Gaststätten und Vereinslokale als Orte der Begegnung) eine zentrale Voraussetzung für unaufwendige und gelingende Unterstützung bleiben wird.

Danksagung Wir danken den Gutachtern für ihre intensive Auseinandersetzung mit unserem Beitrag und die wertvollen Anregungen, Mag. Gerhard Vötsch und Maria Mikulik von der Landentwicklung Steiermark für den offenen Austausch und die Bereitstellung der Daten und den Leitern der ZHN-Gruppen für die Ermöglichung der Einblicke in die lokalen Situationen.

Funding Open access funding provided by University of Natural Resources and Life Sciences Vienna (BOKU).

Open Access Dieser Artikel wird unter der Creative Commons Namensnennung 4.0 International Lizenz (http://creativecommons.org/ licenses/by/4.0/deed.de) veröffentlicht, welche die Nutzung, Vervielfältigung, Bearbeitung, Verbreitung und Wiedergabe in jeglichem Medium und Format erlaubt, sofern Sie den/die ursprünglichen Autor(en) und die Quelle ordnungsgemäß nennen, einen Link zur Creative Commons Lizenz beifügen und angeben, ob Änderungen vorgenommen wurden.

\section{Literatur}

Alisch, M.; Kümpers, S. (2015): Soziale Ungleichheiten in der alternden Gesellschaft - Ein Überblick relevanter Themen und Befunde. In: Informationsdienst Altersfragen 42, 5, 3-11.

ARL - Akademie für Raumforschung und Landesplanung (2016): Daseinsvorsorge und gleichwertige Lebensverhältnisse neu denken. Perspektiven und Handlungsfelder. Hannover. = Positionspapier aus der ARL 108.

Baumgartner, K.; Kolland, F.; Wanka, A. (2013): Altern im ländlichen Raum. Entwicklungsmöglichkeiten und Teilhabepotentiale. Stuttgart.

Blüml, T. (2015): Das Zeit-Hilfs-Netz Steiermark und dessen Beitrag zur Schließung von Versorgungslücken auf kommunaler Ebene. Masterarbeit, Universität für Bodenkultur Wien.

BMASK - Bundesministerium für Arbeit, Soziales und Konsumentenschutz (2015): Bericht zur Lage und zu den Perspektiven des Freiwilligen Engagements in Österreich. 2. Freiwilligenbericht. Wien.

BMVBS - Bundesministerium für Verkehr, Bau und Stadtentwicklung; BBSR - Bundesinstitut für Bau-, Stadt- und Raumforschung (2011): Regionalstrategie Daseinsvorsorge. Denkanstöße für die Praxis. Berlin/Bonn.

BMVI - Bundesministerium für Verkehr und digitale Infrastruktur (Hrsg.) (2015): Anpassungsstrategien zur regionalen Daseinsvorsorge. Empfehlungen der Facharbeitskreise Mobilität, Hausärzte, Altern und Bildung. Berlin. = MORO Praxis 2.

\footnotetext{
18 Aus Sicht der Gruppenleiter kann es gelingen, das Zeit-Hilfs-Netz Steiermark zu einem verlässlich organisierten Netzwerk zu entwickeln. So wäre es beispielsweise interessant, den Mehrwert einer verbesserten Inseratenverwaltung zwecks Erhöhung der Passung von Angebot und Nachfrage auszuloten.
}

Boyle, D. (2014): The Potential of Time Banks to support Social Inclusion and Employability. Luxembourg. = JRC Scientific and Policy Reports EUR 26346 EN. doi: https://doi.org/10.2791/53078

Carroll, J. M. (2013): Co-production Scenarios for Mobile Time Banking. In: Dittrich, Y.; Burnett, M.; Morch, A.; Redmiles, D. (Hrsg.): End-User Development. $4^{\text {th }}$ International Symposium, IS-EUD 2013, Copenhagen, Denmark, June 10-13, 2013. Berlin/Heidelberg, 137-152. doi: https://doi.org/10.1007/978-3-64238706-7_11

Dangschat, J.; Hamedinger, A. (2007): Sozial differenzierte Räume Erkenntnisinteresse, Problemlagen und Steuerung. In: Dangschat, J.; Hamedinger, A. (Hrsg.): Lebensstile, soziale Lagen und Siedlungsstrukturen. Hannover, 206-239. = Forschungs- und Sitzungsberichte der ARL 230.

Dehne, P.; Neubauer, A. (2014): Ländliches Wohnen im Alter, aber wie? Facetten sorgender Gemeinschaften in Mecklenburg-Vorpommern und anderswo. In: Informationsdienst Altersfragen 41, 6, 3-12.

Elsen, S. (2012): Genossenschaften als Organisationen der sozialen Innovation und nachhaltigen Entwicklung. In: Beck, G.; Kropp, C. (Hrsg.): Gesellschaft innovativ - Wer sind die Akteure? Wiesbaden, 85-102. doi: https://doi.org/10.1007/978-3-531-94135-6_5

Fraaß, S.; Görtler, E.; Rosenkranz, D. (2016): Seniorengenossenschaften - Struktur und Erfolgskriterien eines Modells organisierter Solidarität in Deutschland. Ergebnisse der ersten umfassenden Studie im Rahmen eines BMBF-Projektes. In: Informationsdienst Altersfragen 43, 2, 3-11.

Fraňková, E.; Fousek, J.; Kala, L.; Labohý, J. (2014): Transaction network analysis for studying Local Exchange Trading Systems (LETS): Research potentials and limitations. In: Ecological Economics 107, November, 266-275. doi: https://doi.org/10.1016/j. ecolecon.2014.09.009

Glaser, B. G.; Strauss, A. L. (1998): Grounded Theory. Strategien qualitativer Forschung. Bern.

Hiller, H. (2013): DIY: Zeitbanken, Tauschringe, Seniorengenossenschaften - neue alte Selbstgestaltungsräume der Gesellschaft. In: BBSR - Bundesinstitut für Bau-, Stadt- und Raumforschung (Hrsg.): Der demografische Wandel. Eine Gefahr für die Sicherung gleichwertiger Lebensbedingungen? Bonn, 119-127. = BBSR-Online-Publikation 02/2013.

Höllhumer, M.; Trukeschitz, B. (2016): Zeitbanken und Tauschkreise in Österreich. Eine Bestandsaufnahme für 2015. Wien. = Forschungsbericht 1/2016 des Forschungsinstituts für Altersökonomie der Wirtschaftsuniversität Wien, zugleich Forschungsbericht 1/2016 des Projekts CiM.

IFES - Institut für empirische Sozialforschung (2013): Freiwilliges Engagement in Österreich - Bundesweite Bevölkerungsbefragung 2012. Wien.

Kersten, J. (2006): Daseinsvorsorge und demographischer Wandel: Wie ändert sich das Raum- und Staatsverständnis? In: Raumforschung und Raumordnung 64, 4, 245-257. doi: https://doi.org/10. 1007/BF03183174

Lamu, A. N.; Olsen, J. A. (2016): The relative importance of health, income and social relations for subjective well-being: An integrative analysis. In: Social Science \& Medicine 152, 176-185. doi: https://doi.org/10.1016/j.socscimed.2016.01.046

Land Steiermark, Referat Statistik und Geoinformation (2010): Vereine und Wintertourismus in der Steiermark 2009/2010. Graz. = Steirische Statistiken 7/2010.

Land Steiermark, Referat Statistik und Geoinformation (2016a): Regionale Bevölkerungsprognose Steiermark 2015/16 - Bundesland, Bezirke und Gemeinden. Graz. = Steirische Statistiken $5 / 2016$.

Land Steiermark, Referat Statistik und Geoinformation (2016b): Steiermark - Wirtschaft und Konjunktur 2014/15. Graz. = Steirische Statistiken 9/2016. 
Lasker, J.; Collom, E.; Bealer, T.; Niclaus, E.; Young Keefe, J.; Kratzer, Z.; Baldasari, L.; Kramer, E.; Mandeville, R.; Schulman, J.; Suchow, D.; Letcher, A.; Rogers, A.; Perlow, K. (2011): Time Banking and Health: The Role of a Community Currency Organization in Enhancing Well-Being. In: Health Promotion Practice 12, 1, 102-115. doi: https://doi.org/10.1177/1524839909353022

Mayring, P. (2002): Einführung in die Qualitative Sozialforschung. Eine Anleitung zu qualitativem Denken. Weinheim.

Merten, K.; Teipen, P. (1991): Empirische Kommunikationsforschung. Darstellung, Kritik, Evaluation. München.

More-Hollerweger, E.; Sprajcer, S. (2009): Einführung - Definitionen und Abgrenzung von Freiwilligenarbeit. In: BMASK - Bundesministerium für Arbeit, Soziales und Konsumentenschutz (Hrsg.): Freiwilliges Engagement in Österreich - 1. Freiwilligenbericht. Wien, 1-17.

nef - new economics foundation (2008): The new wealth of time: How timebanking helps people to build better public services. London.

Neymotin, F. (2016): Individuals and Communities: the Importance of Neighbors Volunteering. In: Journal of Labor Research 37, 2, 149178. doi: https://doi.org/10.1007/s12122-016-9225-4

Ozanne, L. (2010): Learning to exchange time: Benefits and obstacles to time banking. In: International Journal of Community Currency Research 14, 1-16. doi: https://doi.org/10.15133/j.ijccr.2010.002

Pacione, M. (1997): Local Exchange Trading Systems - A Rural Response to the Globalization of Capitalism. In: Journal of Rural Studies 13, 4, 415-427. doi: https://doi.org/10.1016/S07430167(97)00026-0

Pantea, M.-C. (2015): Understanding non-participation: perceived barriers in cross-border volunteering among Romanian youth. In: International Journal of Adolescence and Youth 20, 3, 271-283. doi: https://doi.org/10.1080/02673843.2013.793205

Peacock, M. S. (2000): Local Exchange Trading Systems. A Solution to the Employment Dilemma? In: Annals of Public and Cooperative Economics 71, 1, 55-78. doi: https://doi.org/10.1111/14678292.00133

Post, M. W. M. (2014): Definitions of Quality of Life: What Has Happened and How to Move On. In: Topics in Spinal Cord Injury Rehabilitation 20, 3, 167-180. doi: https://doi.org/10.1310/sci2003167

Priller, E.; Zimmer, A. (2000): Arbeitsmarkt und Dritter Sektor in Deutschland - $\mathrm{Zu}$ den Ergebnissen des internationalen Vergleichs und einer bundesweiten Organisationsbefragung. In: Zeitschrift für öffentliche und gemeinwirtschaftliche Unternehmen $23,3,304-320$

Schmale I. (2017): Sozialgenossenschaften: Eine wieder entdeckte Rechts- und Wirtschaftsform in der Sozialwirtschaft. In: Schmale I.; Blome-Drees, J. (Hrsg.): Genossenschaft innovativ. Genossenschaften als neue Organisationsformen in der Sozialwirtschaft. Wiesbaden, 11-45. doi: https://doi.org/10.1007/978-3658-11753-5_2
Schmid, T. (2009): Daseinsvorsorge - Möglichkeiten und Grenzen einer lokalen Agenda. In: Kluschatzka, R. E.; Wieland, S. (Hrsg.): Sozialraumorientierung im ländlichen Kontext. Wiesbaden, 7394. doi: https://doi.org/10.1007/978-3-531-91449-7_5

Seyfang, G. (2002): Tackling social exclusion with community currencies: learning from LETS to Time Banks. In: International Journal of Community Currency Research 6, 3, 1-11. doi: https://doi.org/ 10.15133/j.ijccr.2002.002

Seyfang, G. (2003a): Growing Cohesive Communities One Favour at a Time: Social Exclusion, Active Citizenship and Time Banks. In: International Journal of Urban and Regional Research 27, 3, 699706. doi: https://doi.org/10.1111/1468-2427.00475

Seyfang, G. (2003b): 'With a little help from my friends.' Evaluating time banks as a tool for community self-help. In: Local Economy 18, 3, 257-264. doi: https://doi.org/10.1080/0269094032000111 $048 \mathrm{c}$

Shih, P. C.; Bellotti, V.; Han, K.; Carroll, J. M. (2015): Unequal Time for Unequal Value: Implications of Differing Motivations for Participation in Timebanking. In: Proceedings of the 33rd Annual ACM Conference on Human Factors in Computing Systems. Seoul, 1075-1084. doi: https://doi.org/10.1145/2702123.2702560

Simsa, R. (2001): Gesellschaftliche Funktionen und Einflussformen von Nonprofit-Organisationen: eine systemtheoretische Analyse. Frankfurt am Main. $=$ Europäische Hochschulschriften 357.

Slay, J. (2011): More than money. Literature review of the evidence base on Reciprocal Exchange Systems. London.

Statistik Austria (2016a): Ein Blick auf die Gemeinde. http://www. statistik.at/blickgem/index.jsp (18.08.2017).

Statistik Austria (2016b): Altersstruktur der Bevölkerung auf Gemeindeebene. http://statcube.at/statistik.at/ext/statcube/jsf/terms. xhtml (18.08.2017).

Steinführer, A. (2015): Bürger in der Verantwortung. Veränderte Akteursrollen in der Bereitstellung ländlicher Daseinsvorsorge. In: Raumforschung und Raumordnung 73, 1, 5-16. doi: https://doi. org/10.1007/s13147-014-0318-3

Sundeen, R. A.; Raskoff, S. A. (2000): Ports of Entry and Obstacles. Teenagers' Access to Volunteer Activities. In: Nonprofit Management \& Leadership 11, 2, 179-197. doi: https://doi.org/10.1002/ $\mathrm{nml} .11204$

Thorne, L. (1996): Local exchange trading systems in the United Kingdom: A case of re-embedding? In: Environment and Planning A 28, 8, 1361-1376. doi: https://doi.org/10.1068/a281361

Valor, C.; Papaoikonomou, E. (2016): Time Banking in Spain. Exploring their Structure, Management and Users' Profile. In: Revista Internacional de Sociologia 74, 1, e028. doi: https://doi.org/10. 3989/ris.2016.74.1.028

Yamagishi, T.; Cook, K. S. (1993): Generalized Exchange and Social Dilemmas. In Social Psychology Quarterly 56, 4, 235-248. 